\title{
Observation of fluctuation-mediated picosecond nucleation of a topological phase
}

Felix Büttner ${ }^{1 * \ddagger}$, Bastian Pfau $^{2 * \dagger}$, Marie Böttcher ${ }^{3}$, Michael Schneider ${ }^{2}$, Giuseppe Mercurio ${ }^{4}$, Christian M. Günther ${ }^{5,6}$, Piet Hessing ${ }^{2}$, Christopher Klose ${ }^{2}$, Angela Wittmann ${ }^{1}$, Kathinka Gerlinger ${ }^{2}$, Lisa-Marie Kern $^{2}$, Christian Strüber ${ }^{2}$, Clemens von Korff Schmising ${ }^{2}$, Josefin Fuchs ${ }^{2}$, Dieter Engel ${ }^{2}$, Alexandra Churikova $^{1}$, Siying Huang ${ }^{1}$, Daniel Suzuki ${ }^{1}$, Ivan Lemesh ${ }^{1}$, Mantao Huang ${ }^{1}$, Lucas Caretta ${ }^{1}$, David Weder ${ }^{2}$, John H. Gaida ${ }^{7}$, Marcel Möller ${ }^{7}$, Tyler R. Harvey ${ }^{7}$, Sergey Zayko ${ }^{7}$, Kai Bagschik ${ }^{8}$, Robert Carley ${ }^{4}$, Laurent Mercadier $^{4}$, Justine Schlappa ${ }^{4}$, Alexander Yaroslavtsev ${ }^{4}$, Loïc Le Guyarder ${ }^{4}$, Natalia Gerasimova ${ }^{4}$, Andreas Scherz ${ }^{4}$, Carsten Deiter ${ }^{4}$, Rafael Gort ${ }^{4}$, David Hickin ${ }^{4}$, Jun Zhu ${ }^{4}$, Monica Turcato ${ }^{4}$, David Lomidze ${ }^{4}$, Florian Erdinger ${ }^{9}$, Andrea Castoldi ${ }^{10,11}$, Stefano Maffessanti ${ }^{8}$, Matteo Porro ${ }^{4}$, Andrey Samartsev ${ }^{4}$, Jairo Sinova ${ }^{3}$, Claus Ropers $^{7}$, Johan H. Mentink ${ }^{12}$, Bertrand Dupé ${ }^{3 \S}$, Geoffrey S. D. Beach ${ }^{1}$, and Stefan Eisebitt ${ }^{2,5}$

${ }^{1}$ Department of Materials Science and Engineering, Massachusetts Institute of Technology, Cambridge, Massachusetts 02139, USA

${ }^{2}$ Max-Born-Institut, 12489 Berlin, Germany

${ }^{3}$ Institut für Physik, Johannes Gutenberg Universität Mainz, 55099 Mainz, Germany

${ }^{4}$ European XFEL GmbH, 22869 Schenefeld, Germany

${ }^{5}$ Technische Universität Berlin, Institut für Optik und Atomare Physik, 10623 Berlin, Germany

${ }^{6}$ Technische Universität Berlin, Zentraleinrichtung Elektronenmikroskopie (ZELMI), 10623 Berlin, Germany

${ }^{7}$ University of Göttingen, 4th Physical Institute, Göttingen 37077, Germany

${ }^{8}$ Deutsches Elektronen-Synchrotron (DESY), 22607 Hamburg, Germany

${ }^{9}$ Institute of Computer Engineering, Heidelberg University, Germany

${ }^{10}$ Dipartimento di Elettronica, Informazione e Bioingegneria, Politecnico di Milano, Milano, Italy

${ }^{11}$ Istituto Nazionale di Fisica Nucleare, Sezione di Milano, Milano, Italy

${ }^{12}$ Radboud University Nijmegen, Institute for Molecules and Materials, 6525 AJ Nijmegen, Netherlands

*These authors contributed equally to this work.

${ }^{\dagger}$ Corresponding author. E-mail: Bastian.Pfau@mbi-berlin.de.

${ }^{\ddagger}$ Present address: Helmholtz-Zentrum für Materialien und Energie GmbH, 14109 Berlin, Germany

§Present address: Nanomat/Q-mat/CESAM Université de Liège, B-4000 Sart-Tilman, Belgium and Fonds de la Recherche Scientifique (FNRS), Bruxelles, Belgium 
Topological states of matter exhibit fascinating physics combined with intrinsic stability. A key challenge is the fast creation of topological phases, which requires massive reorientation of charge or spin degrees of freedom. Here, we report the picosecond emergence of an extended topological phase comprising many magnetic skyrmions. The nucleation of this phase, followed in real time via single-shot soft $x$-ray scattering after infrared laser excitation, is mediated by a transient topological fluctuation state. This state is enabled by the presence of a time-reversal symmetry breaking perpendicular magnetic field and exists for less than 300 ps. Atomistic simulations indicate that the fluctuation state largely reduces the topological energy barrier and thereby enables the observed rapid and homogeneous nucleation of the skyrmion phase. These observations provide fundamental insights into the nature of topological phase transitions, and suggest a path toward ultrafast topological switching in a wide variety of materials through intermediate fluctuating states.

Femtosecond laser pulses can induce phase transitions and unexpected transient states of matter on ultrafast timescales. Examples range from crystallographic phase transitions ${ }^{1}$ over charge ordering ${ }^{2,3}$ to transitions into hidden quantum phases ${ }^{4}$. If the transitions involve spontaneous symmetry breaking and, moreover, proceed faster than the characteristic quasiparticles can propagate across the transition volume, causality requires the formation of grain-boundary-type topological defects. In non-topological materials, the final-state density of such defects is well understood. For example, the formation of vortices in superfluids and superconductors is described by the Kibble-Zurek mechanism ${ }^{5}$, which links the defect density to a power law of the cooling rate through the second-order phase transition. Moreover, phase transitions exist between bound and free topological states, as formulated in the Berezinskii-Kosterlitz-Thouless (BKT) theory ${ }^{6}$, which however preserve the topological invariants. Phase transitions involving net topological switching, here referred to as global topological phase transitions ${ }^{7}$, are conceptually different and much less understood. In particular, while the annihilation dynamics of a topological state was recently reported ${ }^{8}$, the formation of topological phases has been observed only under quasi-static conditions ${ }^{9-13}$, leaving the mechanism and speed of such phase transitions largely unexplored.

Perpendicular magnetic thin-film ferromagnets are ideal candidates to investigate the dynamics of global topological phase transitions. First, these materials respond strongly to femtosecond laser pulses by ultrafast demagnetization ${ }^{14,15}$ and all-optical switching (AOS) $)^{16}$, even though the mechanism of the latter process is still under debate. Second, many perpendicular magnetic ferromagnets exhibit a topological skyrmion ground 
state in a finite range of externally applied fields ${ }^{17-22}$. The intrinsic stability of such a skyrmion phase arises at room temperature primarily from non-local stray-field interactions while the Dzyaloshinskii-Moriya interaction (DMI) can additionally enforce this stability and determine the skyrmion chirality. The skyrmion phase is topologically protected and therefore often hidden during adiabatic field cycling ${ }^{17,20}$, see Fig. 1a. It can be accessed instead by spatially inhomogenous or dynamical excitations ${ }^{20,23,24}$, including femtosecond laser pulses $^{12,13}$. Due to the large topological energy barriers, which often exceed one hundred times the thermal energy at room temperature ${ }^{25}$, it is expected that the transition into the topological phase is governed by rare, heterogeneous switching events followed by domain wall motion dynamics on a nanosecond timescale or slower ${ }^{12,17}$.

Here, we perform single-shot infrared pump - x-ray probe measurements to follow the topological switching dynamics from a uniform, field-polarized state into a dense skyrmion phase in real time (Fig. 1b). The experiment was conducted at the soft x-ray beamline SCS of the European x-ray free-electron laser source, which provides exceptional sensitivity to the process in combination with temporal and reciprocal-space resolution. We discover that the topological phase transition is essentially completed within $300 \mathrm{ps}$, much faster even than the simple reorientation of existing stripe domains under similar conditions ${ }^{26}$. The unexpected speed of this transition is enabled by evolution through a so far undisclosed transient topological fluctuation state - a state that can be distinguished from non-topological fluctuations ${ }^{27}$ by its evolution into stable, homotopological texture. The high speed is additionally attributed to the homogeneous nucleation of skyrmions, analogous to freezing of supercooled water and the condensation of magnons ${ }^{27}$. We show that the fluctuation state and the skyrmion formation dynamics are in excellent agreement with atomistic spin dynamics simulations, leading to a detailed microscopic understanding of all-optical topological switching.

\section{Demonstration of net topological switching}

Our study of laser-induced topological phase transitions is based on two related perpendicular magnetic multilayer materials: $\mathrm{Ta}(3.6 \mathrm{~nm}) / \mathrm{Pt}(3.7 \mathrm{~nm}) /\left[\mathrm{Pt}(2.7 \mathrm{~nm}) / \mathrm{Co}_{60} \mathrm{Fe}_{20} \mathrm{~B}_{20}(0.9 \mathrm{~nm}) / \mathrm{MgO}(1.5 \mathrm{~nm})\right]_{15} / \mathrm{Pt}(2.7 \mathrm{~nm})$ and $\mathrm{Ta}(3 \mathrm{~nm}) /[\mathrm{Co}(0.6 \mathrm{~nm}) / \mathrm{Pt}(0.8 \mathrm{~nm})]_{15} / \mathrm{Ta}(2 \mathrm{~nm})$, which we subsequently refer to as $\mathrm{Pt} / \mathrm{CoFeB} / \mathrm{MgO}$ and $\mathrm{Pt} / \mathrm{Co}$, respectively. Both materials feature $\mathrm{Pt} / \mathrm{Co}$ interfaces with strong spin-orbit coupling that can, in principle, facilitate chiral interactions and host magnetic skyrmions. To demonstrate topological switching in these materials, we follow the procedure illustrated in Fig. 1a and image the field- and laser-accessible magnetic 
phases at room temperature by in-situ x-ray holography (see Methods and Extended Data Figures 1 and 2 for experimental details).

Figure 2a shows the results for $\mathrm{Pt} / \mathrm{CoFeB} / \mathrm{MgO}$. During field cycling, we find topologically trivial uniform or stripe domain states. We find that these field-induced states can be transformed into pure skyrmion states by single femtosecond laser pulses, provided that time-reversal symmetry is broken by an out-of-plane magnetic field or at least by a significant remanent magnetization of the sample (Fig. 2a, top, and Supplementary Information S.1.). Without such symmetry breaking we observe no skyrmion formation at any laser fluence below the destruction threshold of the material (Fig. 2a, bottom). To demonstrate the topology of the laserinduced magnetic textures, we track their spin-orbit torque current-driven motion, as shown in Fig. 2b and Extended Data Figure 3. We find that all circular textures move in the direction of current and that their trajectories are systematically inclined at an angle to the current axis. This is due to the skyrmion Hall effect $^{28,29}$ and confirms that the observed domains are homochiral skyrmions. These results are consistent with Ref. ${ }^{13}$ and establish the behavior suggested in the main panel of Fig. 1a for chiral materials.

Similar switching characteristics are found in Pt/Co (Fig. 2c), even though thickness-averaged chiral interactions are expected to be negligible in this symmetric material. To determine the chirality and topology of laser-induced circular domains in Pt/Co, we employ Lorentz transmission electron microscopy (L-TEM) instead of current-driven dynamics due to nominally cancelling spin-orbit torques. Circular domains were nucleated in-situ, as described in the Methods, and subsequently imaged by L-TEM at normal incidence. The L-TEM image of the laser-induced state, shown in Fig. 2d, is characterized by a dense arrangement of randomly black and white circles. Unlike the x-ray images, which show the out-of-plane magnetization, L-TEM at normal incidence is sensitive to the Bloch in-plane component of domain walls ${ }^{18,21}$. Specifically, a bright (dark) inner ring corresponds to anticlockwise (clockwise) Bloch chirality (a transport of intensity reconstruction of the in-plane spin configuration is shown in Extended Data Figure 4a). Black and white contrast appears with approximately equal probability. However, this only illustrates that the Bloch chirality, which is not a topological number, is randomly assigned, as expected in this nonchiral material. More importantly, the fact that all circular features, regardless of color, exhibit isotropic L-TEM contrast means that the spins rotate exactly once when moving along the domain perimeter. This implies that, despite the fact that the material itself is nonchiral, and quasistatically-generated spin textures are non-topological (see Extended Data Figure 4b), all laser-nucleated spin textures in our image share the same unit topological charge: they 
are skyrmions.

\section{Signatures of a dynamical phase transition}

Before we study the dynamics of all-optical topological switching, we perform a systematic scan of the parameter space, from which we find two types of switching: thermally activated reversal and phase-transitionmediated switching. These two regimes are illustrated by the evolution of spin textures after consecutive laser pulses, as shown in Fig. 3a. At low fluences, we observe gradual, probabilistic switching, where an initially saturated material is first transformed into a stripe domain state by one or a few laser pulses and where this stripe domain state gradually evolves into a dense skyrmion texture by $>10^{4}$ pulses (see Supplementary Information S.2 for the images corresponding to Fig. 3a). By contrast, above a sharp critical fluence of $\sim 15 \mathrm{~mJ} / \mathrm{cm}^{2}$, we deterministically observe pure skyrmion states after just one laser pulse. This abrupt increase of the switching rate points to a critical behavior, as expected in the vicinity of a (dynamical) phase transition, which we here characterize by the emergence of a global topological charge.

The switching thresholds are similar in both materials (see Supplementary Information S.3), and the same threshold behavior is observed whether starting from a uniform or stripe domain state (Fig. 3b). The threshold is independent of the pulse duration for pulses between $250 \mathrm{fs}$ and $10 \mathrm{ps}$, as demonstrated in Fig. $3 \mathrm{~b}$. The density of skyrmions above the fluence threshold is constant (see Supplementary Information S.2 and S.3). Moreover, the distribution of laser-induced skyrmions, as shown in the inset of Fig. 3a, is characterized by a variance-over-mean of $1.6 \approx 1$, indicative of a shot-noise Poisson distribution with a homogeneous nucleation probability. This contrasts with spin-torque mediated skyrmion nucleation in nominally the same material ${ }^{24}$, where a variance-over-mean of $12.1 \gg 1$ was found in accordance with the fact that spin-torque nucleation relies on extrinsic lateral inhomogeneities to break the symmetry at the point of nucleation ${ }^{24,30}$. See Extended Data Figure 5 and Supplementary Information S.4 for details. Even though the defect density and strength is different for each sample, our observations provide a strong indication that all-optical topological switching is an intrinsic process. Our observations are also in contrast with findings for B20 materials, in which skyrmion nucleation preferentially takes place at sample edges and magnetic phase boundaries ${ }^{12}$. Unlike topological defect formation in non-topological materials ${ }^{2,3,31}$, the pulse-duration-independence of the transition suggests that skyrmion formation above the switching threshold relies on neither the demagnetization time nor the relaxation time to the final state. 


\section{Direct observation of picosecond skyrmion formation dynamics}

We directly followed the dynamics of the global topological phase transition by pulse-resolved small-angle $\mathrm{x}$ ray scattering (SAXS) measurements at the European XFEL, as illustrated in Fig. 1b. These measurements are sensitive to the amplitude squared of the spatial Fourier transform of the out-of-plane magnetization $m_{z}(x, y)$. We employed Pt/Co multilayers for maximum contrast, and we chose the field ( $83 \mathrm{mT}$, see Extended Data Figs. 6-7) and the fluence $\left(\sim 50 \mathrm{~mJ} / \mathrm{cm}^{2}\right)$ in a regime where quasi-static imaging (Fig. $2 \mathrm{c}$ and Supplementary Figure S3) confirms robust switching from a topologically trivial field-polarized state into a pure skyrmion final state. The experiment was conducted in saturate-pump-probe cycles (see Supplementary Information S.7), where each cycle started with a field sweep from $263 \mathrm{mT}$ to $83 \mathrm{mT}$ to prepare the magnetic material in a metastable, uniform initial state, followed by three x-ray pulses and one infrared laser pulse as illustrated in Fig. 1b and Extended Data Fig. 7. The initial state (Fig. 4a) and final state (Fig. 4c) were recorded at every cycle to confirm that we switched from a saturated state to a skyrmion state. For experimental details, see Methods and Supplementary Information S.5-8.

The transient-state SAXS patterns are both quantitatively and qualitatively different from the final state scattering, as illustrated in Fig. 4b. On a timescale of up to $\sim 100$ ps after the laser pulse, we observe weak and almost perfectly uniform scattering. This corresponds to a disordered state characterized by an almost equal distribution of all length scales, similar to recent observations in $\mathrm{GdFeCo}^{27}$ but in our case leading to the formation of a stable texture.

The $q$-integrated instantaneous intensity $I(t)$ is shown in Fig. 4d. This quantity is calculated by (i) summing all detector pixels between $q_{\min }=0.02 \mathrm{~nm}^{-1}$ and $q_{\max } \approx 0.155 \mathrm{~nm}^{-1}$ (see Methods), (ii) normalizing this sum to the incident intensity, and (iii) dividing the result by the average of all final state intensities (also

calculated using steps (i) and (ii)). $I(t)$ is proportional to the coherent $\operatorname{sum} \int_{q_{\min }}^{q_{\max }} \mathrm{d} q\left|\mathcal{F}\left[\sum_{i=1}^{15} m_{z, i}\right]\right|^{2}$ of the lateral Fourier transform of $m_{z}$ in all layers $i$ and therefore a sensitive measure of both the formation of texture in the detected $q$ range and of ferromagnetic alignment between the layers. The measured $I(t)$ exhibits a monotonic increase after the laser excitation. The growth rate is initially small, peaks at $t=(90 \pm 40) \mathrm{ps}$, and subsequently decays exponentially with a time constant of $(56 \pm 9)$ ps (errors are standard deviations, see Methods and Supplementary Information S.9). 300 ps after the laser pulse, the integrated intensity reaches $80 \%$ of the final state intensity. Such strong scattering, irrespective of the missing low- $q$ part, can only occur if $m_{z, i}$ is the same in $\sqrt{0.8}=90 \%$ of all layers. Since the out-of-plane direction is the direction of weakest 
coupling in our sample, we conclude that $300 \mathrm{ps}$ is an upper bound for the timescale at which ferromagnetic order is restored.

Figure $4 \mathrm{e}$ shows the full momentum $q$ and time $t$ resolved scattering $I(q, t)$, where we averaged the azimuthally isotropic scattering data along the azimuth angle. See Extended Data Figure 8 for individual $I(q)$ plots. Around $100 \mathrm{ps}$ the scattering becomes strong enough for quantitative analysis. Here, we already observe a weak peak, corresponding to small skyrmions or reversed domain nuclei that later develop into skyrmions. From the peak position and the decay at its high $q$ shoulder we extract the correlation length (the average distance of skyrmion nuclei) and an estimate for the nuclei size, respectively. Details of this analysis are discussed in the Methods and in Supplementary Information S.10 and the fits are shown in Extended Data Figs. 9-10. The time-dependence of the correlation length and the skyrmion diameter are plotted in Fig. 4d. Both length scales exhibit a rapid increase that initially coincides with an increase of total intensity. However, while the total intensity saturates at $300 \mathrm{ps}$, both the skyrmion size and their average distance continue to increase even at the longest measured delay, $1 \mathrm{~ns}$. Since the average $m_{z}$ does not change after $300 \mathrm{ps,}$ the increase of scattering at low $q$ due to skyrmion growth must be compensated by a reduction of high- $q$ spin fluctuations. This observation indicates that skyrmions grow by condensation of high-energy magnons ${ }^{27}$ and skyrmion coalescence, and not by classical domain wall motion, which contributes to the exceptional high speed of this transition.

\section{Atomistic simulations of topological switching dynamics}

We employ atomistic simulations to qualitatively understand the intrinsic skyrmion nucleation dynamics. Atomistic simulations are the established approach to simulate ultrafast magnetization dynamics because they accurately account for non-equilibrium phenomena and because they can describe the large angles between neighboring spins that typically characterize high-temperature states. To reduce the otherwise overwhelming computational complexity, we consider a simplified model system of a magnetic monolayer and take into account the Heisenberg exchange interaction, the DMI, the easy-axis anisotropy, and the applied field (see Ref. $^{32}$ and Methods for more details). The equilibrium phase diagram of our model system, shown in Fig. 5a, exhibits long-ranged ordered spin-spiral and uniform ferromagnetic states at low temperatures. Above temperature $T_{1}$, fluctuations turn the long-ranged ordered phases into a short-ranged ordered skyrmion phase that is known as the fluctuation-disordered state ${ }^{33,34}$ or the intermediate region ${ }^{35}$. 
In analogy to the experiment, we focus on the nucleation of skyrmions from the FM state, where the initial state holds no information about the chirality. The simulation starts at finite but low temperature at equilibrium in the ordered FM phase. Inspired by the experimentally observed insensitivity for the switching process to details of the excitation, the laser-induced heating is modeled by the generic temperature profile shown in Fig. 5b, i.e., by a sharp increase of the sample temperature $T$ followed by slower cooling (see Methods for details). To compare the simulations with the experiments, we plot the evolution of the squared Fourier transform of the local perpendicular magnetization $m_{z}$ in Fig. $4 \mathrm{f}$. In excellent agreement with the experiment, the simulated scattering shows an initially diffuse distribution that becomes more intense and more concentrated at low $q$ as time progresses. Differences in the absolute values of the time and length scales derive from the simplifications of our model (see Methods). Also note that no meaningful analog of $I(t)$ can be extracted from our simulations since our model comprises only one layer and hence cannot reproduce the reinstatement of interlayer coupling that characterizes the experimental $I(t)$. On a short timescale $(<300 \mathrm{ps}$ in the experiment, $<10 \mathrm{ps}$ in the simulation), however, experiment and simulation exhibit qualitative agreement showing that the simulation is suitable to explain the microscopic mechanism of topological switching in the fluctuation state.

To understand how chirality and topology emerge, we follow the temporal evolution of the fluctuating local topological charge density $q^{ \pm}$, as shown in Fig. 5b. These fluctuations are defined as $q^{ \pm}=(4 \pi)^{-1} \boldsymbol{m}$. $\left(\partial_{x} \boldsymbol{m} \times \partial_{y} \boldsymbol{m}\right)$, which we identify as fractional skyrmion (antiskyrmion) charge densities $q^{+}\left(q^{-}\right)$if the number is positive (negative) ${ }^{35}$. The total charge $Q=\int \mathrm{d} x \mathrm{~d} y\left(q^{+}-q^{-}\right)$must be integer at all times but it does not have to be constant. Bound pairs of $q^{+}$and $q^{-}$with zero net topological charge are generated at all temperatures. However, shortly after the bath has heated to the intermediate region, we additionally observe topological switching, i.e., a deviation of $q^{+}$and $q^{-}$and non-zero $Q$. This is topologically allowed in the intermediate region because enough energy is available for thermally activated switching of a cluster of a few spins $^{35,36}$, as required for skyrmion formation in our high-damping material ${ }^{36}$.

To follow the details of the skyrmion formation process, we plot the time trace of $Q$ and $T$ in Fig. 5c, as well as snapshots of the magnetization pattern and the topological charge density in Figs. 5d-k (see also Supplementary Video S.11). $Q(t)$ exhibits rapid fluctuations on top of a steady increase while $T$ is in the intermediate region. Topological switching events can be identified by a sudden local imbalance of $q^{+}$and $q^{-}$, i.e., by a net local topological charge. This is shown in Figs. $5 \mathrm{~h}-\mathrm{k}$, where we applied a dynamically 
adapted low-pass filter to remove all zero-average thermal fluctuations. The initial low-pass filter cut-off of 8 atomic spacings provides an estimate of the diameter of a skyrmion nuclei in our simulation. Remarkably, skyrmions and antiskyrmions do not form in pairs. Both position and time of creation of skyrmions and antiskyrmions appear uncorrelated. Antiskyrmions are created less frequently and their lifetime is much shorter than of skyrmions. This explains the steady increase of total topological charge of the system. The more the system cools down the less frequent we observe nucleation or annihilation events. Eventually, all switching events stop. At this point, thermal fluctuations only change the skyrmion shape but not their existence. This transition occurs at $\sim 10 \mathrm{ps}$ in the simulation, marking the end of the fluctuation state. In agreement with the experiment, only skyrmions are observed in the final state.

\section{Understanding of the switching dynamics}

Given the combined set of experimental and theoretical results we are in a position to rationalize the mechanism and speed at which the topological charge can emerge. To begin with, our understanding is based on real-space imaging, from which we established that every texture nucleated by high-power laser pulses has the same unit topological charge.

In principle, the appearance of such a skyrmion state shares similarities with the Kibble-Zurek mechanism $(\mathrm{KZM})^{5}$. For example, based on event horizon arguments, the KZM predicts the formation of compact domains by cooling down from the laser-induced paramagnetic phase. However, the actual formation dynamics near the critical point is beyond the scope of KZM. In particular, significant extension of the KZM is required to account for the here-observed transient fluctuation state, the non-trivial temporal evolution of the correlation length during cooling, and the formation of a net global topological charge. In addition, skyrmions can form by Arrhenius activation over the topological energy barrier, as has been evidenced in B20 materials ${ }^{11,12}$. However, the topological nucleation energy barrier ${ }^{25}$ is $8 \pi A t>500 k_{B} T_{300 \mathrm{~K}}$ for each skyrmion (where $A \approx 10 \mathrm{pJ} / \mathrm{m}$ is the exchange stiffness and $t \geq 9 \mathrm{~nm}$ is the magnetic material thickness) and Arrhenius activation over such a large energy barrier must follow entropy-assisted heterogeneous nucleation ${ }^{11,12}$. Both are in contrast with our observations of a large density of skyrmions and a picosecond nucleation timescale.

Our simulations indicate the existence of a new state of matter, the topological fluctuation state, in which the topological energy barrier is effectively eliminated due to massively reduced $A$ and $t$, i.e., a lack of lateral ferromagnetic order and coupling between layers. In momentum space, this state exhibits a characteristic 
evolution of $I(q, t)$ from a broad distribution to a well-defined peak, a feature which is experimentally confirmed. Moreover, the simulations show that in the fluctuation state a high density of topological nucleation centres is possible despite the absence of inhomogeneities in the simulation that would support such localized switching. The simulations show that this switching is enabled by the lack of long-range order in the fluctuation state allowing highly localized dynamics of a few interacting spins, where the reduced excitation volume and the discrete nature of the spins facilitate thermally-induced discontinuous topological switching, a process well beyond the continuum picture of magnonic excitations ${ }^{27}$ (the latter are important though for the subsequent relaxation of the topological nucleation centres to stable skyrmions). The homogeneous nucleation mechanism observed in the simulations is consistent with the largely homogeneous nucleation probability distribution in the experiment, in accordance with the expectation that the exchange-energy-dominated nucleation in the fluctuation state should be mostly insensitive to the much weaker energy scale of local anisotropy and DMI variations that give rise to material heterogeneities responsible for pinning. Moreover, the simulations demonstrate that the total topological charge is stationary slightly before the system leaves the fluctuation state. Hence, a natural upper bound for the timescale at which a global topology emerges is given by the time the system spends in the fluctuation state. Since we cannot directly infer the evolution of the topological charge in the current experiment, we define an upper bound for this time scale from the reinstatement of inter-layer ferromagnetic alignment at $\sim 300 \mathrm{ps}$. The establishment of inter-layer coherence of spin textures in our magnetostatically coupled multilayer material can be taken as an indication that the topological energy barrier has largely recovered, and we therefore conclude that topological switching is completed within this time frame. Further studies are needed to understand if and how a direct quantitative measure of the topological fluctuation state can be obtained.

Two more characteristic features of the topological fluctuation state are required for the formation of a homotopological final state. First, the topological fluctuation state supports short-range spin order, a prerequisite for the stability of a topological nucleus and a key distinction from the paramagnetic phase (even though the paramagnetic phase may be traversed before entering the fluctuation state, as indicated by the lack of an upper fluence bound). And second, a magnetic field must break the time-reversal symmetry, which distinguishes our experiment from previous spatio-temporally resolved experiments in Pt/Co and related materials ${ }^{14-16,26}$. The field ensures that one polarity of skyrmion is preferred over the other and thereby leads to the creation of a net topological charge. Finally, inhomogeneous heating across the sample thickness is also expected to be 
of key importance for symmetric Pt/Co to break the symmetry between skyrmions and antiskyrmions. Similar to compositional gradients in ferrimagnetic materials ${ }^{37}$, the vertical heat gradient due to the finite laser penetration depth may cause transient chiral interactions and thereby induce the required asymmetry that leads to the observed pure skyrmion final state. It will be interesting to investigate this mechanism further by studying the fluence and wavelength dependence of all-optical topological switching.

The picosecond timescale at which the global topological charge emerges is particularly surprising considering that sub-ns switching dynamics is so far only known from ferrimagnets, where it is facilitated by angular momentum transfer between antiparallel sublattices ${ }^{27,38}$. In ferromagnets, by contrast, sub-ns emergence of any magnetic state has only been reported for the recovery of the exact same state as the one before the excitation, i.e., where the dynamics is guided by the magnetic memory of a partially demagnetized material $^{39}$. The formation of new magnetic textures has been observed, for example in CoPd after high fluence laser pulses, but was found to correlate with long remagnetization times of more than $5 \mathrm{~ns}^{26}$. Our results were obtained at a comparable fluence, where full demagnetization of our sample by the laser heat is expected ${ }^{15}$. Still, we find that $300 \mathrm{ps}$ is enough to reach $80 \%$ of the final state magnetic scattering intensity, even though significant parts of the new spin texture are oriented against the previous configuration and against the applied magnetic field. Regardless of topology, such rapid formation of texture is unexpected and underlines that all-optical topological switching is a new form of ultrafast magnetization dynamics.

\section{Outlook}

Using time-resolved $\mathrm{x}$-ray scattering in combination with atomistic spin dynamics simulations, we have demonstrated that femtosecond laser pulses can drive ferromagnetic multilayers into a high-temperature topological fluctuation state in which skyrmions are created by homogeneous nucleation at picosecond timescales. These skyrmions remain stable at room temperature. The here discovered dynamics through a topological fluctuation state is important for the understanding of ultrafast topological phenomena and may not be restricted to magnetic textures. Our work motivates further studies of topological switching in a large variety of systems, which ultimately may disclose the fundamental limits on the speed of topological phase transitions. 


\section{Acknowledgements}

M.B. and B.D. gratefully acknowledge computing time at the Mogon supercomputers. We acknowledge European XFEL in Schenefeld, Germany, for provision of x-ray free-electron laser beamtime at SCS instrument and would like to thank the instrument group and facility staff for their assistance. In particular, we would like to thank Martin Teichmann, Jan Torben Delitz, Alexander Reich, Carsten Broers, Martin Bergemann, Ebad Kamil, Thomas Kluyver, Hans Fanghor, James Moore, Jan Engelke, Markus Kuster, Steffen Hauf, Karsten Hansen, Peter Fischer, Carlo Fiorini, Djelloul Boukhelef, Janusz Szuba, and Krzysztof Wrona for providing the instrumentation and infrastructure that enabled our experiment at the European XFEL. We thank Marek Wieland and Markus Drescher, Universität Hamburg, for providing us their mobile laser hutch for the experiments at DESY. The thank the anonymous referees for the valuable comments that helped us improve our manuscript.

Funding: Work at MIT was supported by the DARPA TEE program. Devices were fabricated using equipment in the MIT Microsystems Technology Laboratory and the MIT Nanostructures Laboratory. The samples were further manufactured at the TU Berlin Nano-Werkbank, which was supported by EFRE under contract number 20072013 2/22. B.P., L.-M.K., K.G., and S.E. acknowledge financial support from the Leibniz Association via the grant No. K162/2018 (OptiSPIN). L.C. acknowledges financial support from the NSF Graduate Research Fellowship Program and from the GEM Consortium. M.B. and B.D. acknowledges financial support from the Alexander von Humboldt Foundation, the Graduate School Materials Science in Mainz, and the Transregional Collaborative Research Center (SFB/TRR) 173 SPIN+X. T.R.H. acknowledges the support of a postdoctoral fellowship from Alexander von Humboldt Foundation. J.H.M. acknowledges funding from the Nederlandse Organisatie voor Wetenschappelijk Onderzoek (NWO) by a VENI grant and the ShellNWO/FOM-initiative "Computational sciences for energy research" of Shell and Chemical Sciences, Earth and Life Sciences, Physical Sciences, FOM and STW.

Author contributions: $\quad$ B.P., F.B., G.S.D.B, and S.E. conceived the study. F.B., C.M.G., M.S., D.E., A.C., I.L., and M.H. fabricated the samples. B.P., F.B., M.S., C.M.G., P.H., C.K., A.W., K.G., L.-M.K., C.S., C.v.K.S., J.F., A.C., S.H., L.C., S.Z., and K.B. performed the experiments at DESY and F.B., B.P., M.S., G.M., C.K., K.G., L.-M.K., S.H., L.C., D.S., R.C., L.M., J.S., A.Y., L.L.G., N.G., A.S., C.D., R.G., D.H., 
J.Z., M.T., and D.L. performed the experiments at XFEL. F.E., A.C., S.M., M.P., and A.S. remotely supported the DSSC detector calibration and operation. J.H.G., M.M., and T.R.H. performed the L-TEM experiments. F.B., B.P., M.S., K.G., P.H., C.K., and A.W. analyzed the x-ray experiments. B.D. and M.B. performed the atomistic modeling with support from J.H.M. F.B., B.P., J.H.M, and B.D. interpreted the results. B.P., F.B., M.S., A.W., K.G., and B.D. prepared the figures and F.B. and J.H.M. wrote the manuscript with input from B.P., B.D., G.S.D.B, and S.E. Supervision by C.R., J.S., G.S.D.B., and S.E. All authors commented on the manuscript.

Competing interests: The authors declare no competing interests.

Data availability: The data represented in Fig. 2 are provided in the Extended Data Figures 2-4. The data represented in Figs. 3-5 are available in zenodo at https: / / doi .org/10.5281/zenodo.3928764. Raw data generated at the European XFEL large-scale facility are available at https://doi .org/10. $22003 /$ XFEL.EU-DATA-002252-00.

Code availability: The data analysis code used in this study is available in zenodo with identifiers https: //doi.org/10.5281/zenodo.3928764. The code for the atomistic simulations is available from the corresponding author on reasonable request.

\section{References}

[1] Wall, S. et al. Ultrafast disordering of vanadium dimers in photoexcited $\mathrm{VO}_{2}$. Science 362, 572-576 (2018).

[2] Vogelgesang, S. et al. Phase ordering of charge density waves traced by ultrafast low-energy electron diffraction. Nature Physics 14, 184-190 (2018).

[3] Zong, A. et al. Evidence for topological defects in a photoinduced phase transition. Nature Physics 15, 27 (2019).

[4] Stojchevska, L. et al. Ultrafast Switching to a Stable Hidden Quantum State in an Electronic Crystal. Science 344, 177-180 (2014). 
[5] Zurek, W. H. Cosmological experiments in condensed matter systems. Physics Reports 276, 177-221 (1996).

[6] Kosterlitz, J. M. \& Thouless, D. J. Ordering, metastability and phase transitions in two-dimensional systems. Journal of Physics C: Solid State Physics 6, 1181-1203 (1973).

[7] Bernevig, B. A., Hughes, T. L. \& Zhang, S.-C. Quantum Spin Hall Effect and Topological Phase Transition in HgTe Quantum Wells. Science 314, 1757-1761 (2006).

[8] Sie, E. J. et al. An ultrafast symmetry switch in a Weyl semimetal. Nature 565, 61-66 (2019).

[9] Yamasaki, Y. et al. Dynamical process of skyrmion-helical magnetic transformation of the chiral-lattice magnet FeGe probed by small-angle resonant soft x-ray scattering. Physical Review B 92, 220421 (2015).

[10] Zhao, X. et al. Direct imaging of magnetic field-driven transitions of skyrmion cluster states in FeGe nanodisks. Proceedings of the National Academy of Sciences 113, 4918-4923 (2016).

[11] Wild, J. et al. Entropy-limited topological protection of skyrmions. Science Advances 3, e1701704 (2017).

[12] Berruto, G. et al. Laser-Induced Skyrmion Writing and Erasing in an Ultrafast Cryo-Lorentz Transmission Electron Microscope. Physical Review Letters 120, 117201 (2018).

[13] Je, S.-G. et al. Creation of Magnetic Skyrmion Bubble Lattices by Ultrafast Laser in Ultrathin Films. Nano Letters 18, 7362-7371 (2018).

[14] Barman, A. et al. Ultrafast magnetization dynamics in high perpendicular anisotropy $[\mathrm{Co} / \mathrm{Pt}]_{n}$ multilayers. Journal of Applied Physics 101, 09D102-09D102-3 (2007).

[15] Pfau, B. et al. Ultrafast optical demagnetization manipulates nanoscale spin structure in domain walls. Nature Communications 3, 1100 (2012).

[16] Lambert, C.-H. et al. All-optical control of ferromagnetic thin films and nanostructures. Science $\mathbf{3 4 5}$, $1337-1340$ (2014). 
[17] Cape, J. A. \& Lehman, G. W. Magnetic Domain Structures in Thin Uniaxial Plates with Perpendicular Easy Axis. Journal of Applied Physics 42, 5732-5756 (1971).

[18] Grundy, P. J. Magnetic bubbles and their observation in the electron microscope. Contemporary Physics 18, 47-72 (1977).

[19] Büttner, F. et al. Dynamics and inertia of skyrmionic spin structures. Nature Physics 11, 225-228 (2015).

[20] Woo, S. et al. Observation of room-temperature magnetic skyrmions and their current-driven dynamics in ultrathin metallic ferromagnets. Nature Materials 15, 501-506 (2016).

[21] Montoya, S. A. et al. Tailoring magnetic energies to form dipole skyrmions and skyrmion lattices. Physical Review B 95, 024415 (2017).

[22] Pollard, S. D. et al. Observation of stable Néel skyrmions in cobalt/palladium multilayers with Lorentz transmission electron microscopy. Nature Communications 8, 14761 (2017).

[23] Romming, N. et al. Writing and Deleting Single Magnetic Skyrmions. Science 341, 636-639 (2013).

[24] Büttner, F. et al. Field-free deterministic ultrafast creation of magnetic skyrmions by spin-orbit torques. Nature Nanotechnology 12, 1040-1044 (2017).

[25] Büttner, F., Lemesh, I. \& Beach, G. S. D. Theory of isolated magnetic skyrmions: From fundamentals to room temperature applications. Scientific Reports 8, 4464 (2018).

[26] Bergeard, N. et al. Irreversible transformation of ferromagnetic ordered stripe domains in single-shot infrared-pump/resonant-x-ray-scattering-probe experiments. Physical Review B 91, 054416 (2015).

[27] Iacocca, E. et al. Spin-current-mediated rapid magnon localisation and coalescence after ultrafast optical pumping of ferrimagnetic alloys. Nature Communications 10, 1756 (2019).

[28] Litzius, K. et al. Skyrmion Hall effect revealed by direct time-resolved X-ray microscopy. Nature Physics 13, 170-175 (2017).

[29] Jiang, W. et al. Direct observation of the skyrmion Hall effect. Nature Physics 13, 162-169 (2016). 
[30] Everschor-Sitte, K., Sitte, M., Valet, T., Abanov, A. \& Sinova, J. Skyrmion production on demand by homogeneous DC currents. New Journal of Physics 19, 092001 (2017).

[31] Eggebrecht, T. et al. Light-Induced Metastable Magnetic Texture Uncovered by in situ Lorentz Microscopy. Physical Review Letters 118, 097203 (2017).

[32] Lemesh, I. et al. Current-Induced Skyrmion Generation through Morphological Thermal Transitions in Chiral Ferromagnetic Heterostructures. Advanced Materials 30, 1805461 (2018).

[33] Janoschek, M. et al. Fluctuation-induced first-order phase transition in Dzyaloshinskii-Moriya helimagnets. Physical Review B 87, 134407 (2013).

[34] Rózsa, L., Simon, E., Palotás, K., Udvardi, L. \& Szunyogh, L. Complex magnetic phase diagram and skyrmion lifetime in an ultrathin film from atomistic simulations. Physical Review B 93, 024417 (2016).

[35] Böttcher, M., Heinze, S., Egorov, S., Sinova, J. \& Dupé, B. B - T phase diagram of Pd/Fe/Ir(111) computed with parallel tempering Monte Carlo. New Journal of Physics 20, 103014 (2018).

[36] Koshibae, W. \& Nagaosa, N. Creation of skyrmions and antiskyrmions by local heating. Nature Communications 5, 5148 (2014).

[37] Kim, D.-H. et al. Bulk Dzyaloshinskii-Moriya interaction in amorphous ferrimagnetic alloys. Nature Materials 18, 685-690 (2019).

[38] Graves, C. E. et al. Nanoscale spin reversal by non-local angular momentum transfer following ultrafast laser excitation in ferrimagnetic GdFeCo. Nature Materials 12, 293-298 (2013).

[39] Kazantseva, N., Nowak, U., Chantrell, R. W., Hohlfeld, J. \& Rebei, A. Slow recovery of the magnetisation after a sub-picosecond heat pulse. EPL (Europhysics Letters) 81, 27004 (2007).

\section{Methods}

Multilayer samples of $\left[\mathrm{Pt}(2.7 \mathrm{~nm}) / \mathrm{Co}_{60} \mathrm{Fe}_{20} \mathrm{~B}_{20}(0.9 \mathrm{~nm}) / \mathrm{MgO}(1.5 \mathrm{~nm})\right]_{15}$ were grown by Ar magnetron sputter deposition onto silicon nitride membranes with a seed layer of $\operatorname{Ta}(3.6 \mathrm{~nm}) / \mathrm{Pt}(3.7 \mathrm{~nm})$ and capped with $\operatorname{Pt}(2.7 \mathrm{~nm})$. The Pt layers were grown at an Ar pressure of $3.5 \mathrm{mTorr}$. For all other layers, the Ar pressure was 
3 mTorr. In this material stack, a sizable Dzyaloshinskii-Moriya interaction on the order of $1.5 \mathrm{~mJ} / \mathrm{m}^{2}$ was previously reported ${ }^{32}$, leading to homochiral left-handed Néel-type spin textures throughout the film thickness $^{40} . \mathrm{Ta}(3 \mathrm{~nm}) /[\mathrm{Co}(0.6 \mathrm{~nm}) / \mathrm{Pt}(0.8 \mathrm{~nm})]_{15} / \mathrm{Ta}(2 \mathrm{~nm})$ multilayers were grown on $150 \mathrm{~nm}$ thick $\mathrm{Si}_{3} \mathrm{~N}_{4}$ membranes by DC magnetron sputtering (RF magnetron sputtering in case of Ta) at $2.7 \times 10^{-3}$ mbar Ar pressure and $6.5 \times 10^{-9} \mathrm{mbar}$ base pressure. Magnetic tracks of $10 \mu \mathrm{m}$ width were fabricated by electron-beam lithography and lift-off, followed by focused ion beam (FIB) milling to reduce the track widths to the dimensions shown in the figures. The tracks were contacted with $\mathrm{Ti}(5 \mathrm{~nm}) / \mathrm{Au}(100 \mathrm{~nm})$ pads made by a two-step process of electron-beam lithography and lift-off followed by shadow-mask deposition. The holography masks on the back side of the membranes comprised a $[\mathrm{Cr}(5 \mathrm{~nm}) / \mathrm{Au}(55 \mathrm{~nm})]_{20}$ multilayer into which the object hole and the reference holes were milled by FIB. Scanning electron micrographs of all our devices are shown in Extended Data Figure 2.

X-ray imaging and laser excitation were performed via Fourier-transform x-ray holography in a maskbased approach ${ }^{41}$ at beamline P04 at PETRA III in Hamburg, Germany. We used circularly polarized x-rays at the $\mathrm{L}_{3}$ resonance of $\mathrm{Co}(778 \mathrm{eV})$ to obtain magnetic contrast via the $\mathrm{x}$-ray magnetic circular dichroism. Magnetic contrast was obtained from helicity difference images, while sum images show transmission contrast reflecting topography in our samples (see Extended Data Figure 1). We chose to reconstruct the x-ray transmission amplitude instead of the magnetic phase contrast since amplitude images are more robust against contrast fluctuations in our setup (in which holograms were acquired with a central beamstop). Linearly polarized laser pulses with a wavelength of $1030 \mathrm{~nm}$ were generated by an Amplitude Satsuma fiber laser with software-controlled pulse duration, power, repetition rate, and number of pulses. The laser was coupled into the vacuum using an upstream on-axis mirror. The laser pulse energy $(E)$ and laser focus were calibrated at the position of the sample immediately after the experiment. The laser spot on the sample followed a Gaussian profile with an elliptical footprint of $\sigma_{x}=40 \mu \mathrm{m}$ and $\sigma_{y}=30 \mu \mathrm{m}$ (long and short axes, respectively; standard deviation). The incident laser fluence $(I)$ was estimated as peak fluence of the laser spot: $I=E /\left(2 \pi \sigma_{x} \sigma_{y}\right)$. The overlap of X-ray and IR beams was adjusted in-situ using a fluorescent screen and a microscope. Stability of the laser position on the sample was confirmed by observing the same switching threshold at the beginning and at the end of a measurement series. After establishing a stable switching threshold, we used the switching threshold to calibrate the fluence and to compare measurement series of same samples between different beamtimes. The error in fluence due to these beam position uncertainties is estimated to be $10 \%$. 
The process of reconstructing magnetic images via x-ray holography is illustrated in Extended Data Figure 1. We first record holograms with positive and negative helicity light. The setup to record holograms is discussed in detail in the literature ${ }^{41,42}$. By subtracting the two helicity holograms from each other we isolate the magnetic contribution. An inverse Fourier transform yields the Patterson map, which includes the cross-correlations between the specimen transmission function and the delta-like reference transmission functions. These cross correlations are the reconstructions of the magnetic images, convolved with the reference beam profile. Finally, we propagate the reconstruction to the position along the beam axis where the reference is smallest, hence providing the sharpest image ${ }^{43}$. The final result is the image shown in Extended Data Figure 1f. All images in the main text were reconstructed in this way.

Lorentz transmission electron microscopy was performed at the Göttingen Ultrafast Transmission Electron Microscope (UTEM), a modified JEOL 2100F allowing for in-situ optical sample excitation ${ }^{31,44}$. The microscope was operated at $200 \mathrm{kV}$ and in a low magnification mode, in which the main objective lens is normally current-free and can be used to apply an arbitrary out-of-plane magnetic field at the sample, ranging from zero to several hundred mT. Images where acquired using a CEOS Energy-Filtering and Imaging Device (CEFID) - filtering out inelastically scattered electrons with an energy loss larger than $6 \mathrm{eV}$ - and a TVIPS XF416. Magnetic contrast was obtained in overfocus imaging conditions ${ }^{45}$.

The skyrmion state was prepared by initially saturating the sample in an out-of-plane magnetic field of $250 \mathrm{mT}$, then reducing the field to $25 \mathrm{mT}$ and exciting the sample with an optical burst of laser pulses at a wavelength of $770 \mathrm{~nm}$. The burst consisted of 20 pulses each with a fluence of $8 \mathrm{~mJ} / \mathrm{cm}^{2}$ at $610 \mathrm{kHz}$ repetition rate and was used to compensate for the smaller maximum achievable fluence of the setup.

Atomistic simulations were carried out on a square lattice in a volume of $100 \times 100 \times 1$ atomic spacings (unit cells). Periodic boundary conditions were employed in $x$ and $y$ directions. We have considered the Heisenberg Hamiltonian

$$
H=-\sum_{i j} J_{i j}\left(\mathbf{m}_{i} \cdot \mathbf{m}_{j}\right)-\sum_{i j} \mathbf{D}_{i j} \cdot\left(\mathbf{m}_{i} \times \mathbf{m}_{j}\right)-\sum_{i} K m_{i, z}^{2}-\sum_{i} \mu_{0} M_{s}\left(m_{i, z} B_{z}\right) .
$$

Here, $i, j$ enumerate lattice sites and $\mathbf{m}_{i}=\mathbf{M}_{i} / M_{s}$ are unit vectors along the local spin direction and $m_{i, z}$ is the out-of-plane component. The model parameters are: atomic spin moments $M_{s}=4.66 \mu_{B}$, first nearest neighbor exchange interaction $J_{1}=1.5 \mathrm{meV}$ (zero otherwise), and easy axis anisotropy $K=0.46 \mathrm{meV}$ along the out-of-plane direction. The Dzyaloshinskii-Moriya interaction vectors $\mathbf{D}_{i j}$ are in-plane and perpendicular to the spin pair position vector $\mathbf{r}_{i j}$, favoring Néel-type spin textures. The magnitude of $\mathbf{D}_{i j}$ is $D_{1}=0.6 \mathrm{meV}$ 
for nearest neighbors and zero otherwise. $B_{z}$ is the variable external out-of-plane magnetic field. Note that in our experimental multilayer material, non-collinear textures, such as stripe domains and skyrmions, are stabilized primarily by non-local stray field interactions ${ }^{25}$. To compensate for the fact that our model, due to its computational complexity, does not include stray field interactions, we have increased the values of $D / J$ and $D / K$ compared to typical values of monolayer-thick films ${ }^{35}$ to a value where the $B=0, T=0$ state resembles the experimentally observed stripe domain state. See Ref. ${ }^{32}$ for details.

Magnetization dynamics simulations follow the Landau-Lifschitz-Gilbert (LLG) equation

$$
\frac{\mathrm{d} \mathbf{m}_{i}}{\mathrm{~d} t}=-\gamma \mathbf{m}_{i} \times \mathbf{B}_{i}+\alpha \mathbf{m}_{i} \times \frac{\mathrm{d} \mathbf{m}_{i}}{\mathrm{~d} t}-\gamma \mathbf{m}_{i} \times \boldsymbol{\zeta}(t),
$$

where $\mathbf{B}_{i}$ is the effective magnetic field at spin $i, \gamma=1.76 \times 10^{11} \mathrm{As} / \mathrm{kg}$ is the gyromagnetic ratio, and $\alpha=0.3$ is the Gilbert damping. This equation was numerically solved in time steps of $1 \mathrm{fs}$ using a Heun solver (and cross checked with an Euler solver, both giving the same result). At each time step, the internal field is determined from the Hamiltonian Eq. (1) as

$$
\mathbf{B}_{i}=-\frac{1}{M_{s}} \frac{\partial H}{\partial \mathbf{m}_{i}}
$$

The dynamics simulations were performed in an external field of $B_{z}=2.05 B_{c}$, where $B_{c}=0.12 \mathrm{~T}$ defines the transition from a spin spiral to the ferromagnetic phase at low temperature. The stochastic field $\zeta(t)$ describes the coupling of the magnetic system to the finite temperature fluctuations of the non-magnetic degrees of freedom of the sample. At each time, all Cartesian components $\nu$ of this field are randomly generated according to a Gaussian probability distribution with zero mean:

$$
\left\langle\zeta^{\nu}(t)\right\rangle=0
$$

By the fluctuation-dissipation theorem, the spatial (lattice sites $i, j$ ) and temporal correlations between the different components $\nu, \kappa$ of the stochastic field are determined by the sample temperature $T$ and the dimensionless damping parameter $\alpha$ via:

$$
\left\langle\zeta_{i}^{\nu}(t) \zeta_{j}^{\kappa}\left(t^{\prime}\right)\right\rangle=2 \alpha \frac{k_{B} T}{\gamma M_{s}} \delta_{i, j} \delta_{\nu, \kappa} \delta\left(t-t^{\prime}\right)
$$

We used the following function to model the temporal evolution of the sample temperature:

$$
T(t)= \begin{cases}T_{0} & \text { if } t<t_{0} \\ T_{0}+I_{0} \exp -\frac{\left(t-t_{0}-t_{M}\right)^{2}}{\sigma^{2}} & \text { if } t_{0}<t<t_{1} \\ T_{0}+\frac{I_{1}}{\left(t-t_{0}-t_{m}\right)^{k}} & \text { if } t_{1}<t\end{cases}
$$


where $t_{0}=0$ ps defines the beginning of the laser pulse, $t_{1}=2.6 \mathrm{ps}, t_{M}$ corresponds to the maximum peak of intensity $I_{0}$ and mean height width $\sigma$. The cooling down is modeled by an inverse polynomial of power $k$ and of intensity $I_{1}$. The data presented in Fig. 5 of the main paper corresponds to $I_{0}=0.309 T_{c}, I_{1}=0.209 T_{c}$, $t_{M}=2.2 \mathrm{ps}, t_{m}=1.7 \mathrm{ps}, \sigma=0.7 \mathrm{ps}$ and $k=0.54$, a temperature profile which is inspired by typical excitation curves of the electron temperature system by a femtosecond laser pulse ${ }^{46}$. The generic choice is motivated by the experimentally observed robustness of the nucleation process to the laser fluence, i.e., to the amplitude and shape of the applied heat pulse. The temperature $T_{0}$ was chosen to start the simulation in the FM phase $T_{0}=0.307 T_{c}$. All temperatures are given in units of the Curie temperature, which is $T_{c}^{\exp }=650 \mathrm{~K}$ in $\mathrm{Pt} / \mathrm{CoFeB} / \mathrm{MgO}^{32}$ and $T_{c}=29 \mathrm{~K}$ in the simulation. This low simulation value has no absolute meaning other than helping us to reduce the computation time. For similar reasons we have used a faster cooling time than in the experiments, leading to the discrepancies in absolute timescales.

Monte Carlo simulations were carried out to obtain the phase diagram presented in Fig. 5a. Parallel tempering Monte Carlo was employed to overcome possible local minima created by metastable states such isolated skyrmions and antiskyrmions. We have simulated 180 different replicas whose temperature were uniformly distributed between $1 \mathrm{~K}$ and $60 \mathrm{~K}$. The different replicas were swapped 2000 times and $10^{6}$ autocorrelation steps were used between each swap. The Curie temperature is $T_{c}=29 \mathrm{~K}$. The starting configuration was chosen as the ferromagnetic or the spin spiral ground state at $T=0 \mathrm{~K}$ depending on the magnetic field $B_{z}$.

Low pass filtering of the topological charge density. To apply the low-pass filter, we multiplied the simulated charge density distributions in reciprocal space with a circular binary mask. The mask was convolved with a Gaussian (standard deviation of 2 reciprocal unit cells) to reduce ringing artefacts in the real-space maps. We used the fast Fourier transform algorithm to numerically transform the two-dimensional real-space charge densities to reciprocal space and back. For a mask radius of $r$ (in reciprocal unit cells), this effectively averages out all fluctuations with a real-space periodicity smaller than $1 / r$. For the data shown in Figs. $5 \mathrm{~h}-\mathrm{j}$, a single reciprocal unit cell has a size of $1 / 100$ (atomic spacings) ${ }^{-1}$. Thus, the utilized mask radius of 12 reciprocal unit cells removes fluctuations with real-space periodicities shorter than $100 / 12 \approx 8$ atomic spacings. The specific value of 12 reciprocal unit cells was chosen as the value that best highlights the position of the skyrmions and antiskyrmions during the fluctuation state (Figs. 5i,j). Note that we know the total number of skyrmions minus the total number of antiskyrmions from the integrated topological charge (Fig. 5c). The 
optimum filter radius changes towards later times and has been adjusted to 4 reciprocal unit cells in Fig. 5k. For display in Fig. 5, the filtered data was first normalized to the same total topological charge as before filtering and then rescaled by a factor $1 /\left(\pi r^{2}\right)$ in order to account for the low-pass filter blurring.

Time-resolved small-angle $x$-ray scattering measurements of all-optical topological switching were conducted at beamline SCS at the European X-ray Free-Electron Laser (European XFEL). The $[\mathrm{Pt} / \mathrm{Co}]_{15}$ magnetic materials (see Sample Fabrication) was grown on stoichiometric (high-stress) $\mathrm{Si}_{3} \mathrm{~N}_{4}$ membranes because these membranes remain flat even after material deposition, as confirmed by optical inspection. Therefore, the only remaining source of scattering was the magnetic texture of the sample. The membrane size was $200 \mu \mathrm{m} \times 200 \mu \mathrm{m}$, much larger than the $\mathrm{x}$-ray spot ( $30 \mu \mathrm{m}$ in diameter, full width at half maximum). The infrared laser beam (wavelength $1030 \mathrm{~nm}$ ) was focused to a spot size of $\sigma_{x}=142 \mu \mathrm{m}$ and $\sigma_{y}=120 \mu \mathrm{m}$ (Gaussian standard deviation along $x$ and $y$, respectively), leading to a homogeneous fluence of $\sim 50 \mathrm{~mJ} / \mathrm{cm}^{2}$ within the x-ray spot size (estimated by measuring the laser power before the vacuum incoupling mirror). Spatial overlap of the infrared laser and the x-ray beam were measured by a camera image of both beams on an x-ray fluorescent pyrolytic boron nitride screen at the sample position, which exhibits visible light fluorescence upon x-ray illumination and also diffusely reflects the infrared beam. The temporal overlap (time zero) was adjusted in two steps. Coarse time zero was determined by measuring the photo-current of the FEL and optical pulses at the conductive tip of a coaxial cable. Fine temporal overlap was measured using the specular reflectivity of the infrared beam at a $\mathrm{Si}_{3} \mathrm{~N}_{4}$ surface, which transiently decreases when the $\mathrm{Si}_{3} \mathrm{~N}_{4}$ crystal is exposed with $\mathrm{x}$-rays ${ }^{47}$.

The x-ray beam was monochromatized to the photon energy of maximum Co absorption at $778.9 \mathrm{eV}$ ( $\sim 240 \mathrm{meV}$ band width) using the second order of the monochromator to suppress higher harmonics. The beam was circularly polarized by transmission through a perpendicular magnetic TbCo film, even though the polarization has no relevance for the results presented here. The intensity of every x-ray pulse after the monochromator was measured using a gas absorption monitor and the result was used to normalize all detected scattering patterns. These scattering patterns were recorded by a DSSC detector ${ }^{48,49} 1.44 \mathrm{~m}$ downstream of the sample. The detector had $1024 \times 1024$ pixels, arranged in 16 ladders of $128 \times 512$ hexagonal pixels with a cartesian spacing of $236 \mu \mathrm{m}$ and $204 \mu \mathrm{m}$ in $x$ and $y$ direction, respectively ${ }^{50}$. We applied a mask to remove parasitic infrared light and defective pixels from the recorded data, as discussed in detail in Supplementary Information S.6. The detector position was chosen such that the intense transmitted and 
forward scattered x-ray beam at and close to $q=0$ is centered on a gap between the detector ladders. All data were recorded by first saturating the sample at $263 \mathrm{mT}$ and subsequently reducing the field to $83 \mathrm{mT}$, where a train of three x-ray pulses and one infrared laser pulse was applied (see Fig. 1b). Before and after each transient and final state x-ray pulse we recorded additional dark frames, i.e., six camera frames in total per train. Due to communication issues with the magnet power supply, not all x-ray pulse trains were acquired in this sequence. We applied several filters to select the correct trains with statistically meaningful information, as described in detail in Supplementary Information S.7. Moreover, we determined the threshold for x-ray induced skyrmion nucleation and attenuated the incoming beam to well below that threshold, see Supplementary Information S.5 and Extended Data Fig. 6. The data acquisition and analysis were performed using the Karabo infrastructure of the European XFEL ${ }^{51}$.

All data was corrected by subtracting dark frames, i.e., data recorded after the experiment without x-ray or infrared light. Furthermore, we subtracted the average of the dark frames before and after the transient and final state from the respective state. All scattering patterns were normalized to the incident x-ray intensity. In addition, the scattering patterns in Figs. $4 \mathrm{a}-\mathrm{c}$ were normalized to the maximum counts in the final state image (Fig. 4c). $I(t)$ in Fig. 4d was obtained by integration between $q_{\min }=0.021 \mathrm{~nm}^{-1}$ and $q_{\max } \approx 0.155 \mathrm{~nm}^{-1}$. The same ranges were applied to transient and final state integrals. The lower integration limit was given by the central gap and the upper limit was introduced because higher $q$ data was found to contribute many pixels with high noise level and were therefore excluded from this plot. The time-dependent intensity was fit with a logistic function $I(t)=L /\left(1+\exp \left(-\left(t-t_{0}\right) / \tau_{\text {eq }}\right)\right)$ to extract the equilibration time constant $\tau_{\text {eq }}$. To estimate the error of the fit parameters, $q_{\max }$ was varied between $0.08 \mathrm{~nm}^{-1}$ and $0.255 \mathrm{~nm}^{-1}$ and the standard deviations of the resulting arrays of normalized intensities and logistic fit values are reported as error bars, see Supplementary Information S.9. Note that this is justified because the particular choice of $q_{\max }$ determines the magnitude of the total intensity in Fig. $4 \mathrm{~d}$ but not the functional shape and the equilibration time constant and the saturation beyond $300 \mathrm{ps}$ are robustly obtained for $q_{\max }$ in the given range.

The data in Fig. 4e was obtained by averaging the intensity in rings of constant momentum transfer around the centre (i.e., by integrating the intensity and dividing by the number of contributing pixels). Fig. $4 \mathrm{f}$ shows the similarly averaged squared Fourier transform of the $m_{z}(x, y, t)$ maps shown in Fig. $5 \mathrm{~d}-\mathrm{g}$, which represents the anticipated x-ray scattering patterns of the simulation. The experimental data has lower noise compared to the simulations because the area probed by the x-ray pulse greatly exceeds the simulation area. 
To correct for a slow drift of the dark counts of the camera, we subtracted the initial state in Figs. 4d,e before normalizing to the average peak intensity of final state scattering (see Supplementary Information S.8).

The correlation length and the estimate for skyrmion diameter were determined from the peak position $q_{\text {peak }}$ in the $I(q)$ spectra and a Guinier fit of the high- $q$ shoulder of the peak ${ }^{52}$. The Guinier fit parameter is proportional to the mean diameter of the scattering particles. The proportionality factor depends on the shape and density of the particles. We estimate this factor using the known average size of skyrmions in the final state, as determined from Fig. 2 (see size histogram in Supplementary Figure S11). The remaining error of the final state fits is hence only an error of the linear regression. The error of the skyrmion diameter in the transient state has been estimated as $30 \%$ of the diameter value, based on the $q$ range to which we have applied the Guinier fit ${ }^{52}$. The correlation length was determined based on a local parabolic fit of $q_{\text {peak }}$ (more complex fitting with a Voigt function was found to yield the same results). The error bars of the correlation length in Fig. $4 \mathrm{~d}$ represent the fit error of $q_{\text {peak }}$. The procedure and fit results are discussed in detail in Supplementary Information S.10.

For the data and code underlying the figures in this paper and in the Supplementary Information, see Ref. ${ }^{53}$.

[40] Litzius, K. et al. The role of temperature and drive current in skyrmion dynamics. Nature Electronics 3, 30-36 (2020).

[41] Eisebitt, S. et al. Lensless imaging of magnetic nanostructures by X-ray spectro-holography. Nature 432, 885-888 (2004).

[42] Büttner, F. Dynamic Imaging with X-ray Holography. In Naydenova, I., Babeva, T. \& Nazarova, D. (eds.) Holographic Materials and Optical Systems (InTech, 2017).

[43] Geilhufe, J. et al. Achieving diffraction-limited resolution in soft-X-ray Fourier-transform holography. Ultramicroscopy 214, 113005 (2020).

[44] Feist, A. et al. Ultrafast transmission electron microscopy using a laser-driven field emitter: Femtosecond resolution with a high coherence electron beam. Ultramicroscopy 176, 63-73 (2017).

[45] Schneider, M., Hoffmann, H. \& Zweck, J. Lorentz microscopy of circular ferromagnetic permalloy nanodisks. Applied Physics Letters 77, 2909-2911 (2000). 
[46] Koopmans, B. et al. Explaining the paradoxical diversity of ultrafast laser-induced demagnetization. Nature Materials 9, 259-265 (2010).

[47] Krupin, O. et al. Temporal cross-correlation of x-ray free electron and optical lasers using soft x-ray pulse induced transient reflectivity. Optics Express 20, 11396-11406 (2012).

[48] Porro, M. et al. Development of the DEPFET Sensor With Signal Compression: A Large Format X-Ray Imager With Mega-Frame Readout Capability for the European XFEL. IEEE Transactions on Nuclear Science 59, 3339-3351 (2012).

[49] Hansen, K. et al. Qualification and Integration Aspects of the DSSC Mega-Pixel X-Ray Imager. IEEE Transactions on Nuclear Science (2019).

[50] Erdinger, F. et al. The DSSC pixel readout ASIC with amplitude digitization and local storage for DEPFET sensor matrices at the European XFEL. In 2012 IEEE Nuclear Science Symposium and Medical Imaging Conference Record (NSS/MIC), 591-596 (2012).

[51] Fangohr, H. et al. Data Analysis Support in Karabo at European XFEL. In TUCPA01, International Conference on Accelerator and Large Experimental Control Systems, 245-252 (JACOW, Geneva, Switzerland, 2018).

[52] Feigin, L. A. \& Svergun, D. I. Structure Analysis by Small-Angle X-Ray and Neutron Scattering (Plenum Press, New York, NY, USA, 1987).

[53] Büttner, F. et al. Data and code for Observation of fluctuation-mediated picosecond nucleation of a topological phase (2020). URL https: / / zenodo.org/record/3928764. 

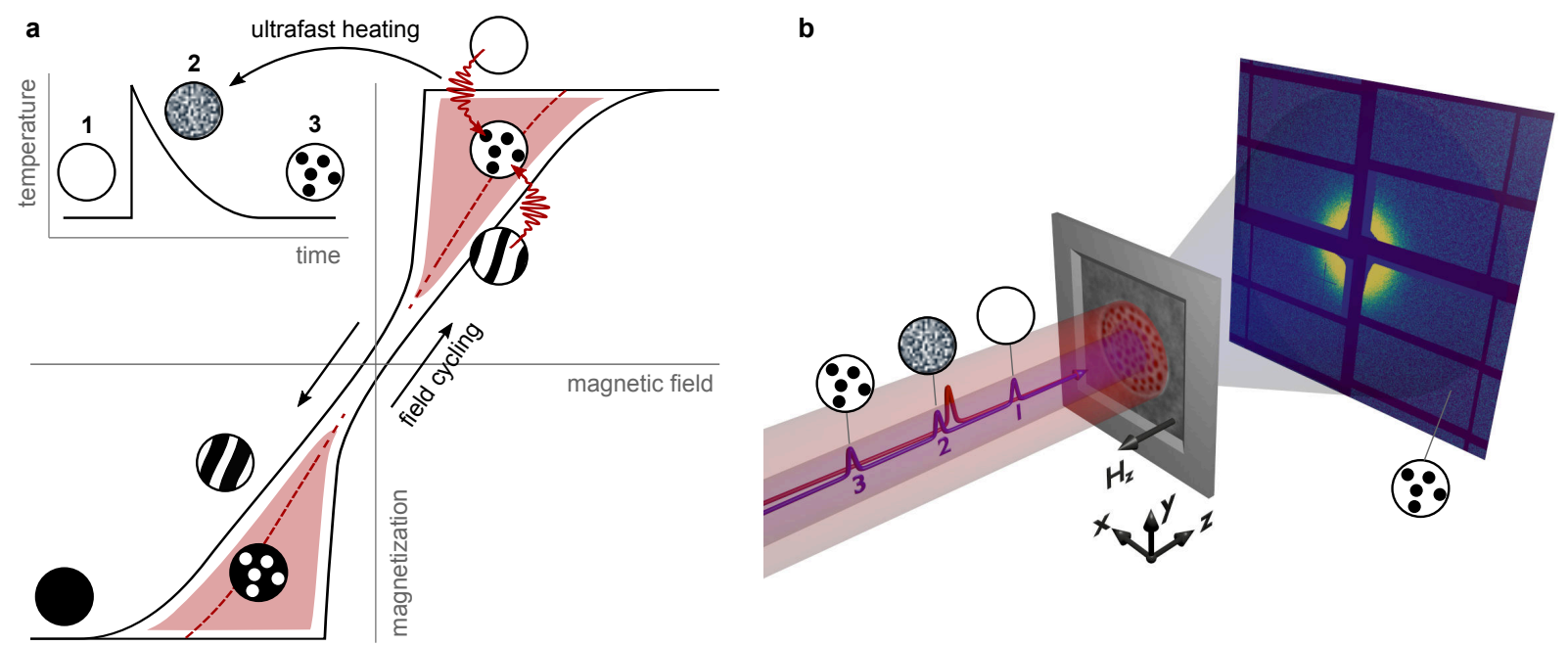

Figure 1. Topological magnetic phase transition mediated by a transient fluctuation state. (a) Typical roomtemperature phases of a ferromagnetic thin-film skyrmion material as a function of perpendicular magnetic field. Field-accessible magnetic phases are indicated by pictographs along the solid black hysteresis curve (homogeneous black or white: uniform down or up, respectively; stripes: stripe domains; circles: skyrmions). The laser-accessible skyrmion phase is located between the hysteresis branches. The dashed red line schematically indicates the average out-of-plane magnetization of the lowest energy skyrmion texture. Wavy red arrows illustrate phase transitions induced by laser pulses. The inset illustrates the evolution of sample temperature and magnetic states from a uniform state (1) via a fluctuation state (2) to a skyrmion texture (3). (b) Schematic of the time-resolved detection process. The sample comprises a magnetic multilayer on an x-ray transparent membrane in an out-of-plane magnetic field $H_{z}$. The sample is exposed to a train of three x-ray pulses and one infrared laser pulse, which has a well-defined delay with respect to the second x-ray pulse. The coherent diffraction of the x-ray pulses is recorded by a camera with submicrosecond readout speed. The scattering pattern shown here corresponds to a skyrmion state. 

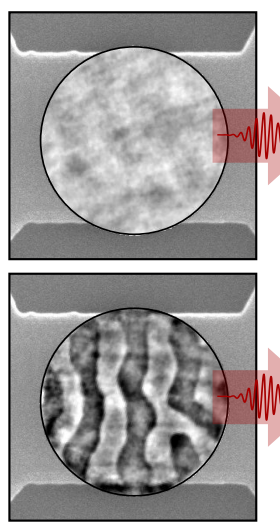

b

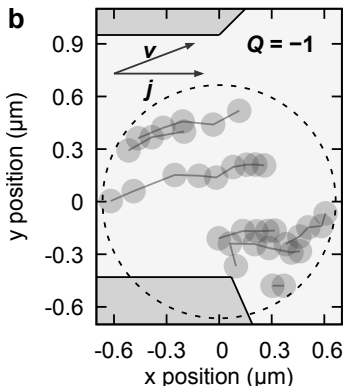

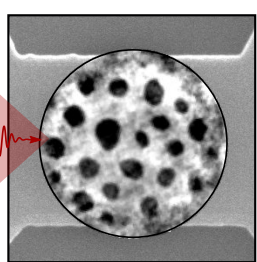
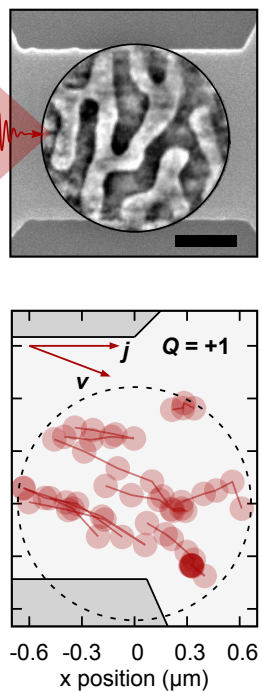

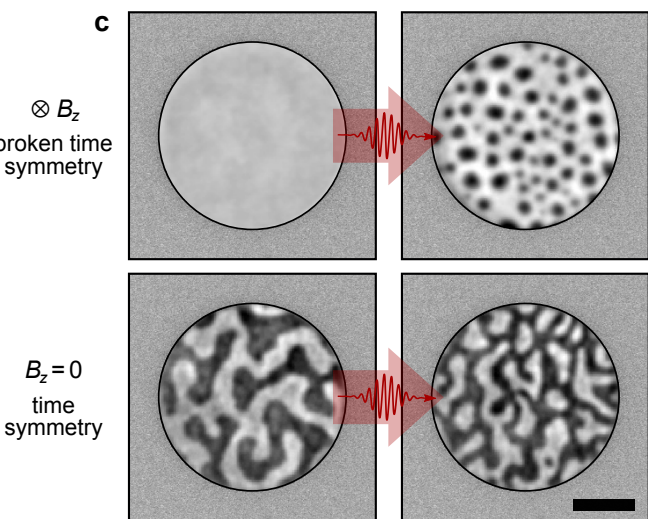

d

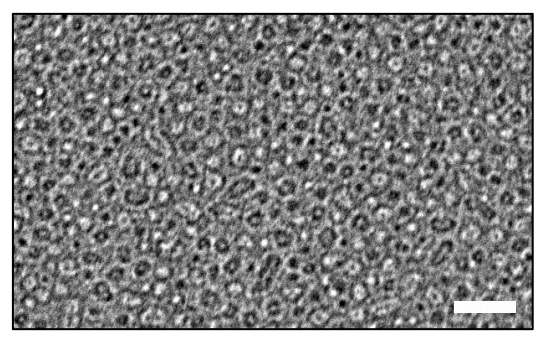

Figure 2. All-optical topological switching in $\mathrm{Pt} / \mathrm{CoFeB} / \mathrm{MgO}$ and Pt/Co. (a) Single-shot laser-induced phase transitions in $\mathrm{Pt} / \mathrm{CoFeB} / \mathrm{MgO}$ at variable external perpendicular magnetic field (top: following the sequence $1 \rightarrow 3$ illustrated in Fig. 1a at $B_{z}=19 \mathrm{mT}$; bottom: after AC out-of-plane demagnetization). The background shows scanning electron micrographs of the respective sample. See Extended Data Fig. 2 for larger SEM images. The gray contrast of the images in the circles represents the out-of-plane magnetization $m_{z}$. The switching fluence was $16 \mathrm{~mJ} / \mathrm{cm}^{2}$. (b) Trajectories of laser-nucleated skyrmions in $\mathrm{Pt} / \mathrm{CoFeB} / \mathrm{MgO}$ driven by spin-orbit current pulses $j$ in a positive field ( $37 \mathrm{mT}$, left) and negative magnetic field $(-36 \mathrm{mT}$, right). See Extended Data Figure 3 for all frames. The dark-gray background indicates areas outside the magnetic track. The dashed circle marks our field of view. Full circles correspond to skyrmion positions and the lines connect positions of the same skyrmion in subsequent frames. Position error bars are smaller than the data points. The topology $Q$ is confirmed by the angle of the trajectory $v$ with respect to the current direction $j$. (c) Same data as in (a) but for Pt/Co (top: following the sequence $1 \rightarrow 3$ illustrated in Fig. 1a at $B_{z}=41 \mathrm{mT}$ and a fluence of $17 \mathrm{~mJ} / \mathrm{cm}^{2}$; bottom: after AC out-of-plane demagnetization and a fluence of $65 \mathrm{~mJ} / \mathrm{cm}^{2}$ ). (d) Lorentz transmission electron micrograph of laser-nucleated skyrmions in Pt/Co in a field of $25 \mathrm{mT}$ using overfocus imaging condition. Bright (dark) contrast inside a circular texture indicates anticlockwise (clockwise) Bloch chirality. Scale bars, $500 \mathrm{~nm}$. 

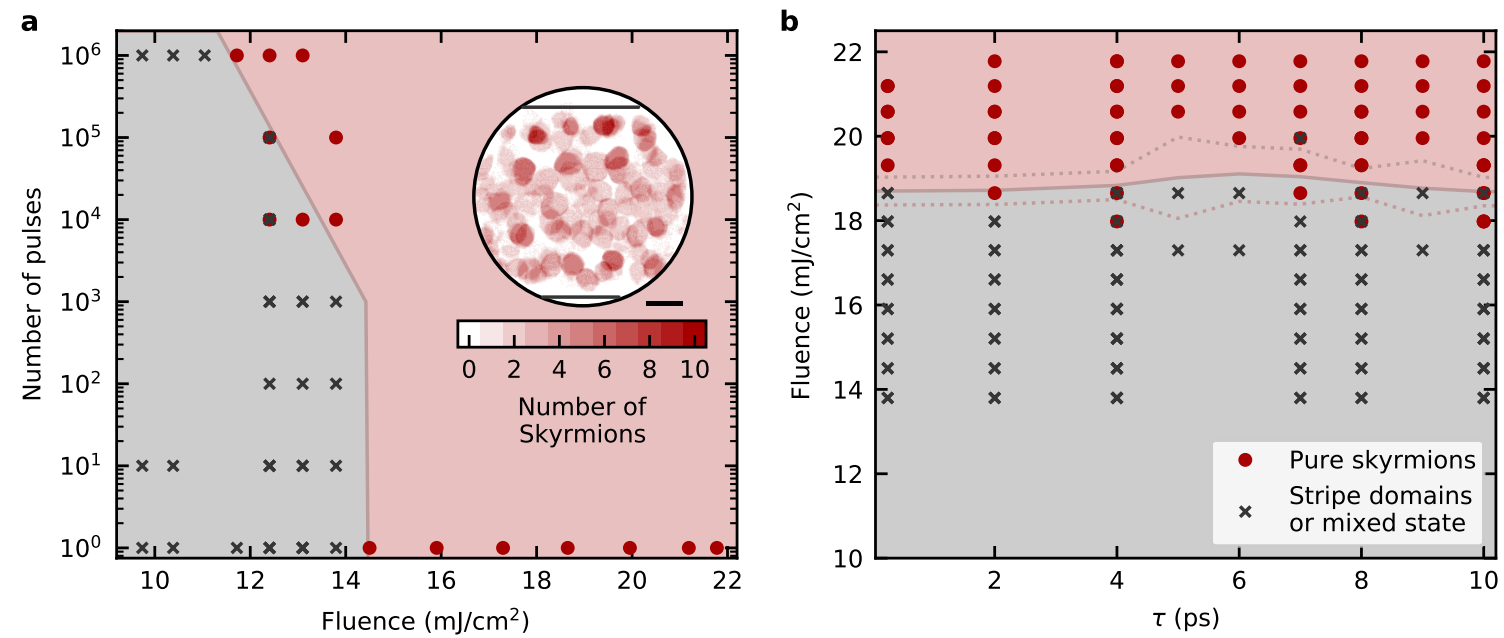

Figure 3. Switching threshold in $\mathrm{Pt} / \mathrm{CoFeB} / \mathrm{MgO}$ as a function of laser pulse number, fluence, and duration. (a) Final state as a function of laser fluence and number of laser pulses, starting from a saturated state (for the corresponding real-space images, see Fig. S2). Mixed states are observed below $\sim 14.5 \mathrm{~mJ} / \mathrm{cm}^{2}$, where they gradually transform into skyrmion arrays by many pulses. As pure skyrmion states we identify patterns of fully enclosed domains with a density that remains constant upon subsequent laser exposure. All data correspond to $\tau=250 \mathrm{fs}$ pulses and an applied field of $\mu_{0} H_{z} \approx 10 \mathrm{mT}$. The background color is a guide to the eye. The inset shows the integrated distribution of skyrmions in ten patterns obtained by single laser pulses from a stripe domain state. Scale bar, $250 \mathrm{~nm}$. (b) Final state after one single laser pulse as a function of pulse duration $\tau$ and fluence at $\mu_{0} H_{z} \approx 16 \mathrm{mT}$, starting from a stripe domain state. The dashed lines denote the uncertainty interval separating the high-fluence single-shot skyrmion states from the mixed states observed at lower fluences (std. dev.). 

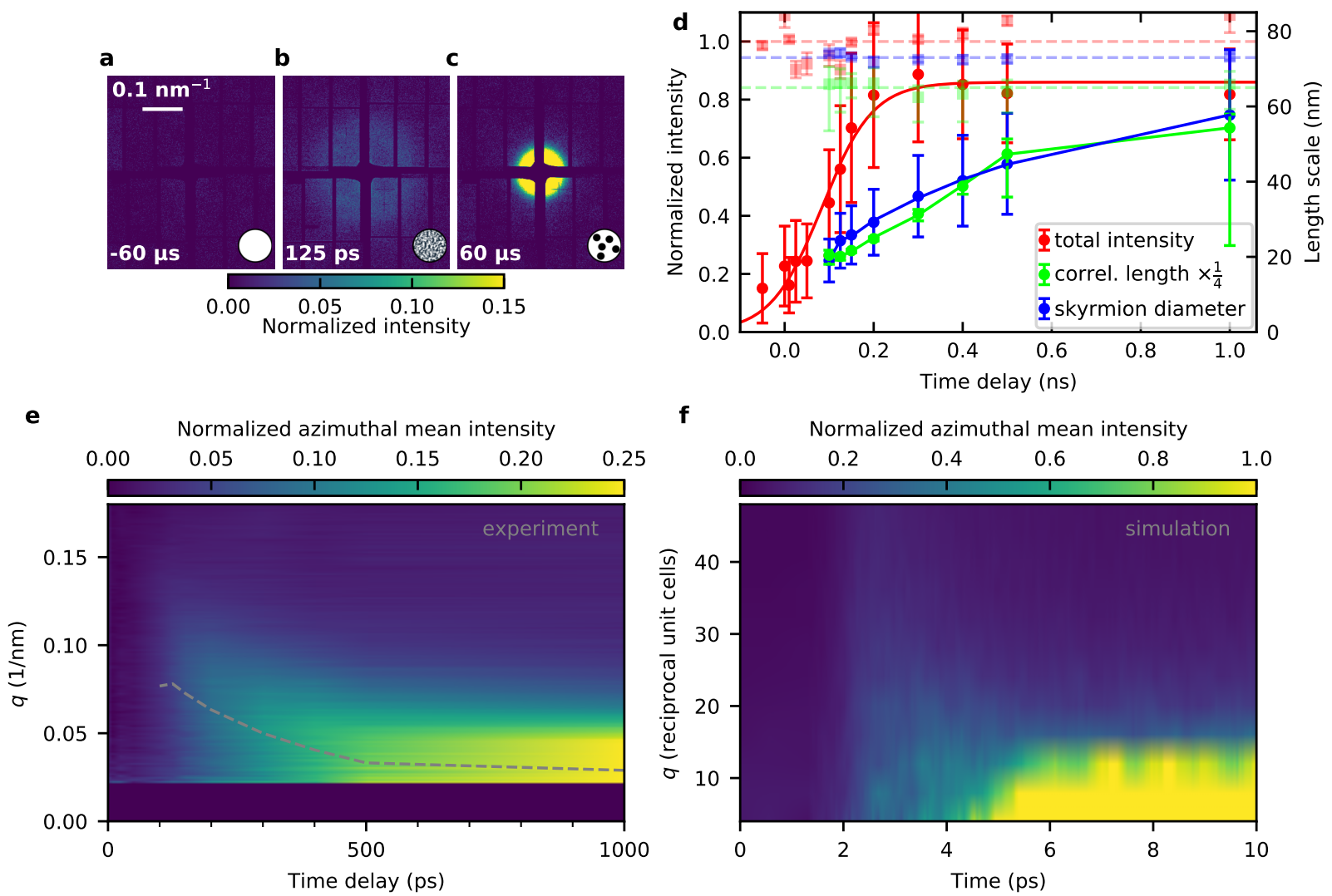

Figure 4. Time-resolved observation of the topological phase transition. (a-c) Example small-angle x-ray scattering patterns before (a), during (b), and after (c) the laser-induced nucleation dynamics (cropped to the central region). The delay between the respective $\mathrm{x}$-ray pulse and the infrared pulse is indicated. The insets depict the corresponding real-space spin textures. (d) Normalized total scattered intensity, correlation length, and skyrmion diameter as a function of time delay (circular data points). Square data points show the control measurements of the final state for each pump-probe shot. The solid red line is a fit to a logistic function, see Methods for details. Other solid lines are guides to the eye. Dashed lines represent the average of the final state values. Error bars represent estimated standard error of the mean. Error bars on intensity were estimated by variation of the upper integration limit, see Methods. Other error bars are fit and model errors, see Supplementary Information S.10 for details. (e) $q$-dependent scattering (azimuthal average) of the transient state as a function of pump-probe delay. Intensity $I$ at larger $q$ values corresponds to higher spatial gradients in real space. The dashed line indicates the fitted peak positions. This line only seems to be at the low $q$ side of the maximum due to the broad and asymmetric shape of $I(q)$ at small time delays. (f) Simulated $q$-dependent scattering, corresponding to the data shown in Fig. 5. 

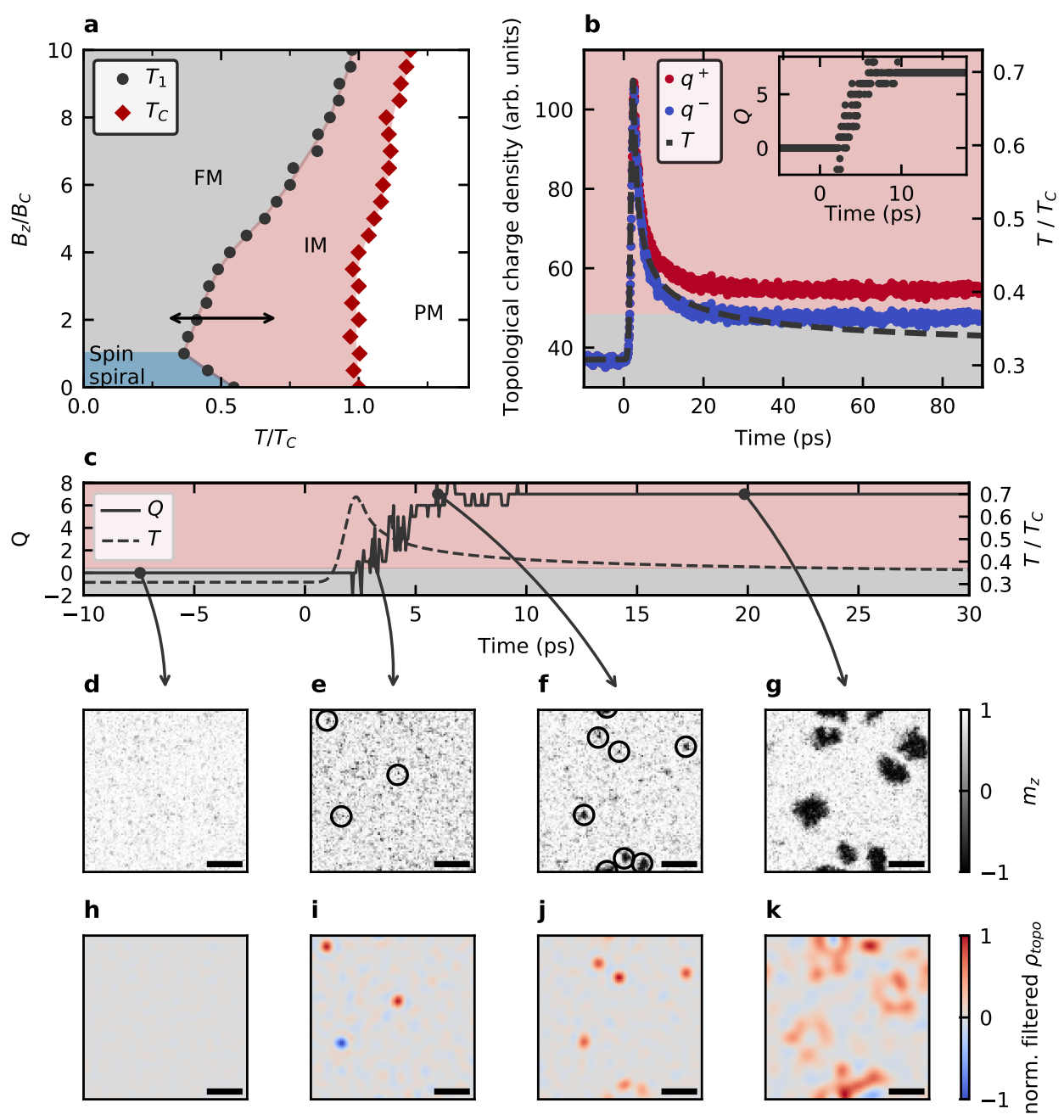

Figure 5. Atomistic simulation of laser-induced skyrmion nucleation. (a) Static phase diagram of our model. $T / T_{c}$ : reduced temperature with the Curie temperature $T_{c}=29 \mathrm{~K} ; B_{z} / B_{c}$ : reduced out-of-plane magnetic field with $B_{c}=0.12 \mathrm{~T}$; FM: ferromagnetic phase; IM: intermediate region; PM: paramagnetic phase. (b) Simulated laser heating and cooling sequence (also illustrated by the double-headed arrow in (a)). The black dashed curve shows the heat-bath temperature $T$. The colored background indicates the static FM and IM phases that would form if the temperature was varied adiabatically. The density of skyrmion (red) and antiskyrmion (blue) fractional topological charges, as defined in the text, is plotted on the same timescale. The inset shows the total integrated topological charge $Q=\int \mathrm{d} x \mathrm{~d} y\left(q^{+}-q^{-}\right)$of the entire simulation area. (c) Magnification of the evolution of $Q$ and $T$. (d-g) Snapshots of the normalized out-of-plane magnetization $m_{z}$ at selected times, as indicated in (c). (h-k) Snapshots of the topological charge density $\rho_{\text {topo }}$ at the same times. A low-pass filter was applied to separate the agglomerated integer topological charges (skyrmions and antiskyrmions) from the high-frequency thermal fluctuations of zero-average fractional charges and normalized to the absolute maximum (see Methods for details). The thereby detected positions of skyrmions and antiskyrmions are indicated by circles in the magnetization maps. Scale bars, 20 atomic spacings. 

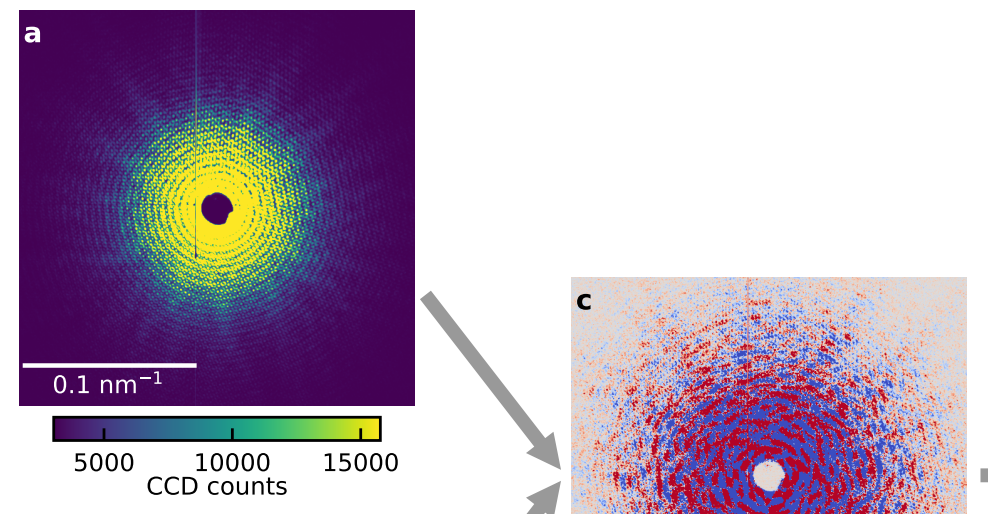

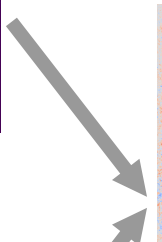

c

b

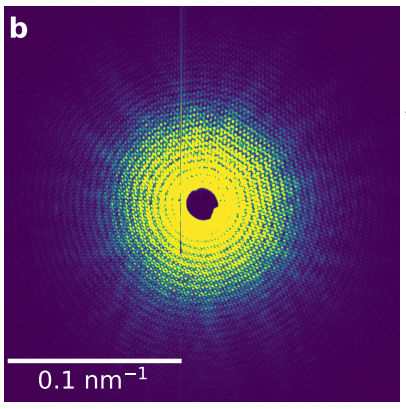

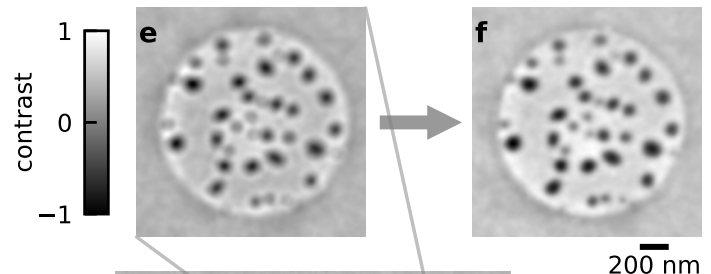

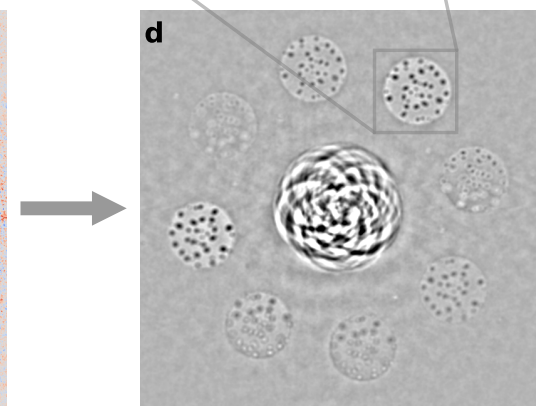

$20 \overline{\mathrm{nm}}$

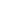

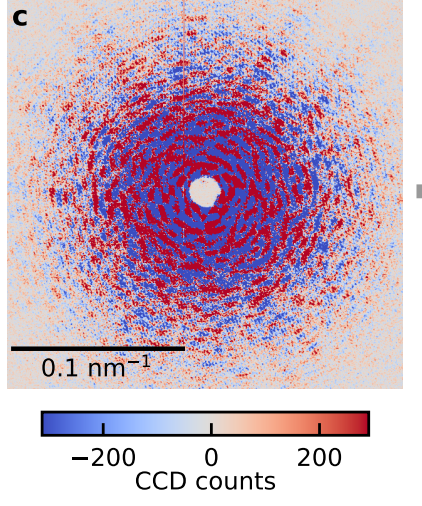

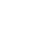



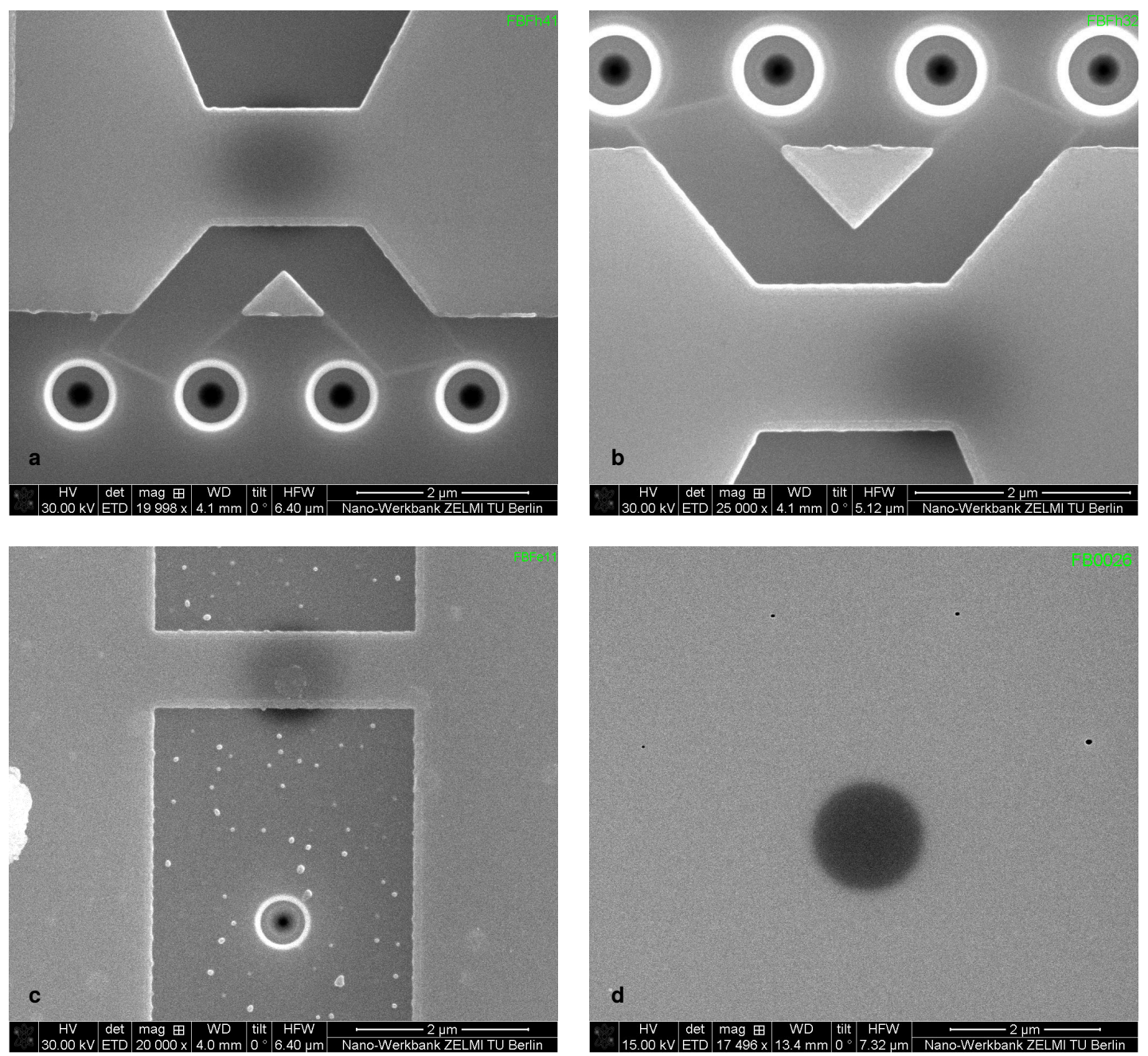

Extended Data Figure 2. Scanning electron micrographs of the holography samples employed in this study. (ac) $\mathrm{Pt} / \mathrm{CoFeB} / \mathrm{MgO}$ samples with patterned tracks for current injection. The holographic field of view (FOV) is defined by an aperture in the $\mathrm{Cr} / \mathrm{Au}$ mask on the opposite sample side and is visible as shadow behind the tracks. The reference pinholes (four pinholes in (a) and (b), one pinhole in (c)) have their smallest exit aperture also at the opposite sample side, i.e., the mask side. The sample in (a) was used for Figs. 2a and 3, sample in (b) for Fig. 2b, and the sample in (c) for Supplementary Information S.1. (d) Pt/Co sample with continuous magnetic film. The FOV appears as a shadow approximately in the center of the image and is surrounded by four reference pinholes with their smallest exit on the Pt/Co film side. The sample was used for Fig. 2c. 
a

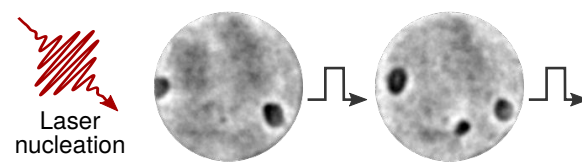

$\odot$

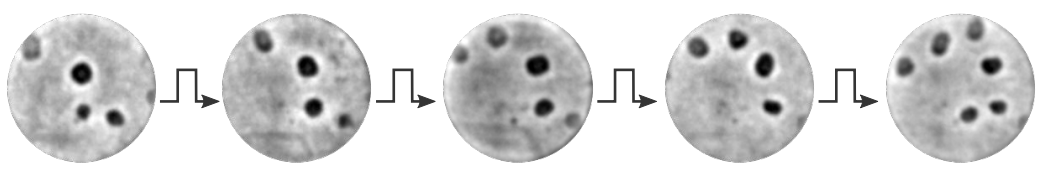

b
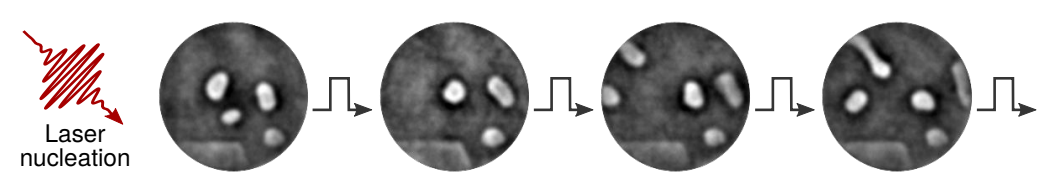

$\bigotimes$

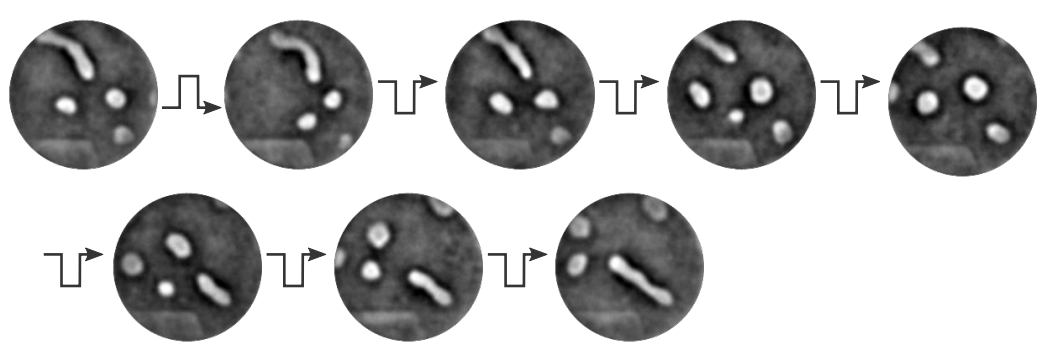

c

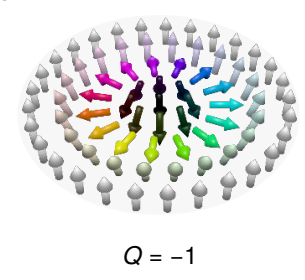

d

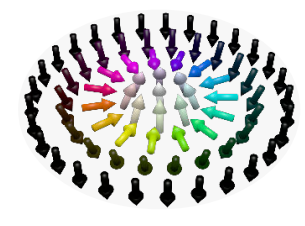

$Q=1$

Extended Data Figure 3. Measurement of the skyrmion topology via the skyrmion Hall effect. (a) For $B_{z}=$ $37 \mathrm{mT}$. (b) For $B_{z}=-36 \mathrm{mT}$. The first image in each sequence was produced by a single $>16 \mathrm{~mJ} / \mathrm{cm}^{2}$ laser pulse from a saturated state. Between subsequent images, single current pulses of the indicated polarity and direction ( $4 \mathrm{~ns}$ duration and between $7 \times 10^{11} \mathrm{~A} / \mathrm{m}^{2}$ and $9 \times 10^{11} \mathrm{~A} / \mathrm{m}^{2}$ in amplitude) were applied. The size of all circles is $1.3 \mu \mathrm{m}$. (c,d) Spin structures of a left-handed (black in x-ray images) and right-handed (white) skyrmion, respectively. 

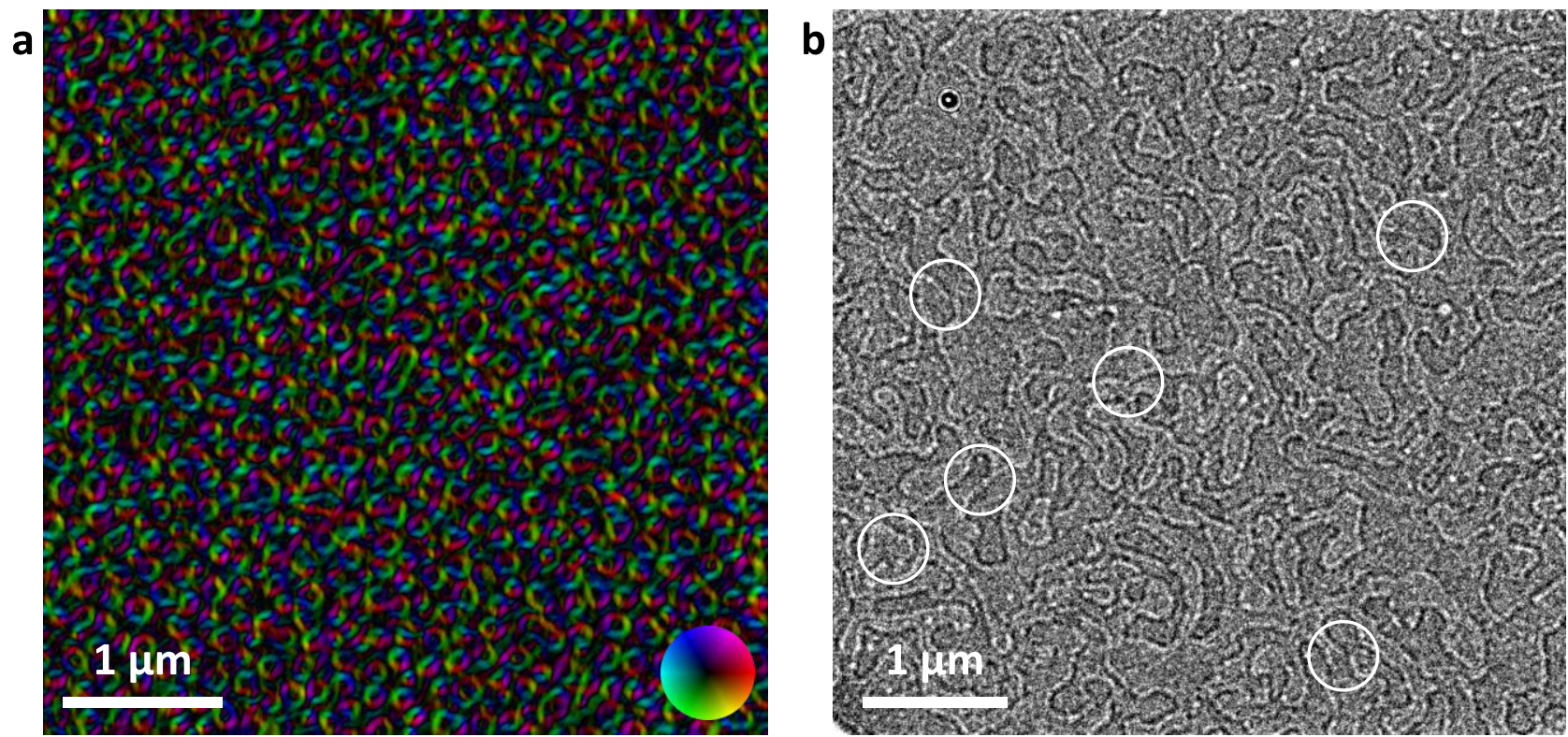

Extended Data Figure 4. Lorentz transmission electron (L-TEM) micrographs of Pt/Co. (a) Transport of intensity reconstruction Fig. $2 d$ of the main paper. The color shows in-plane orientation of magnetization, as indicated by the color wheel. (b) Representative L-TEM image of a stripe domain state at zero field obtained by adiabatic field cycling. White circles highlight vertical Bloch lines (note that the signal-to-noise ratio is not sufficient in this case to perform the TIE analysis). Both images were recorded in overfocus conditions ${ }^{45}$. 
a

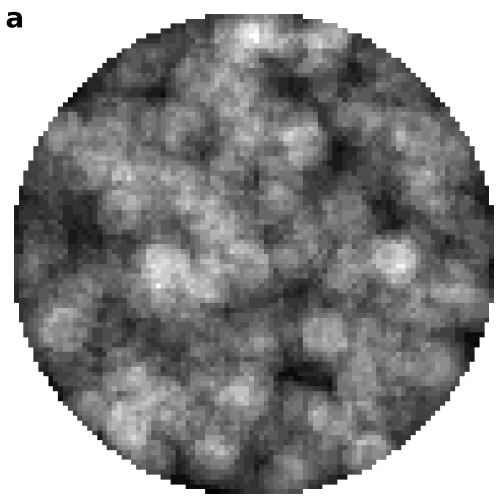

homogeneous probability var $/$ mean $=1.151$

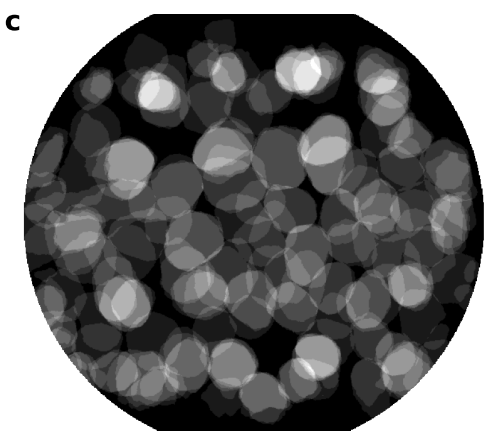

laser nucleated skyrmions $\mathrm{var} / \mathrm{mean}=1.6$

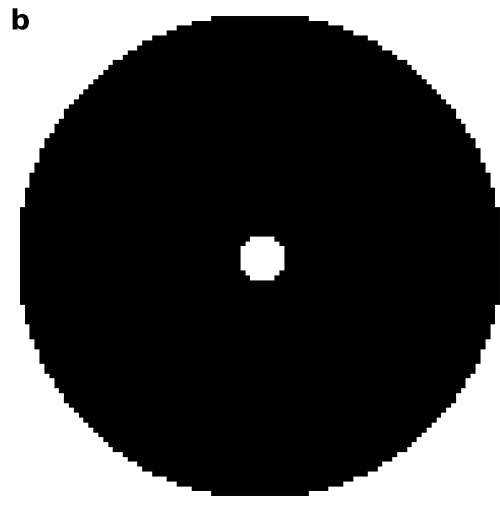

inhomogeneous probability var/mean $=1002.9$

d

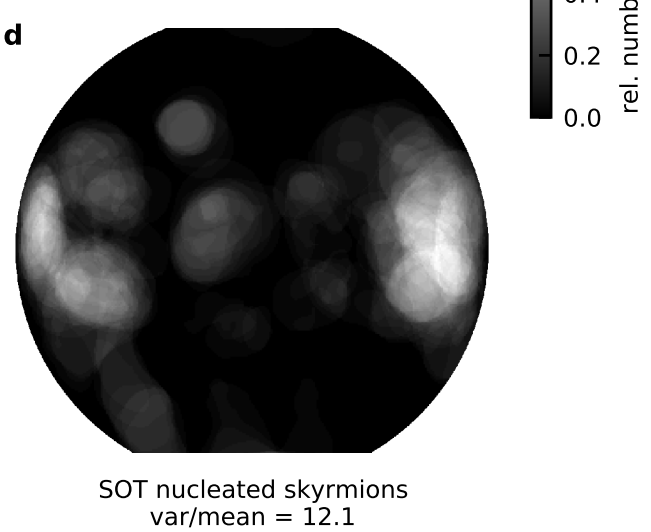

Extended Data Figure 5. Simulated and experimentally observed spatial distribution of nucleated skyrmions.

(a) Simulated integrated skyrmion count of 1000 skyrmions (10 px diameter) distributed according to a homogeneous nucleation probability in a $100 \mathrm{px}$ diameter field of view. (b) 1000 skyrmions distributed with $100 \%$ probability in the central pixel. (c) Experimentally observed distribution of optically-nucleated skyrmions (reproduced from Fig. 3 in the main text). (d) Experimentally observed distribution of spin-orbit torque nucleated skyrmions (reproduced from ${ }^{24}$ ). The horizontal diameters of the fields of view are $100 \mathrm{px}$ in (a) and (b), $1490 \mathrm{~nm}$ in (c) and $900 \mathrm{~nm}$ in (d). See Supplementary Information S.4 for details. 


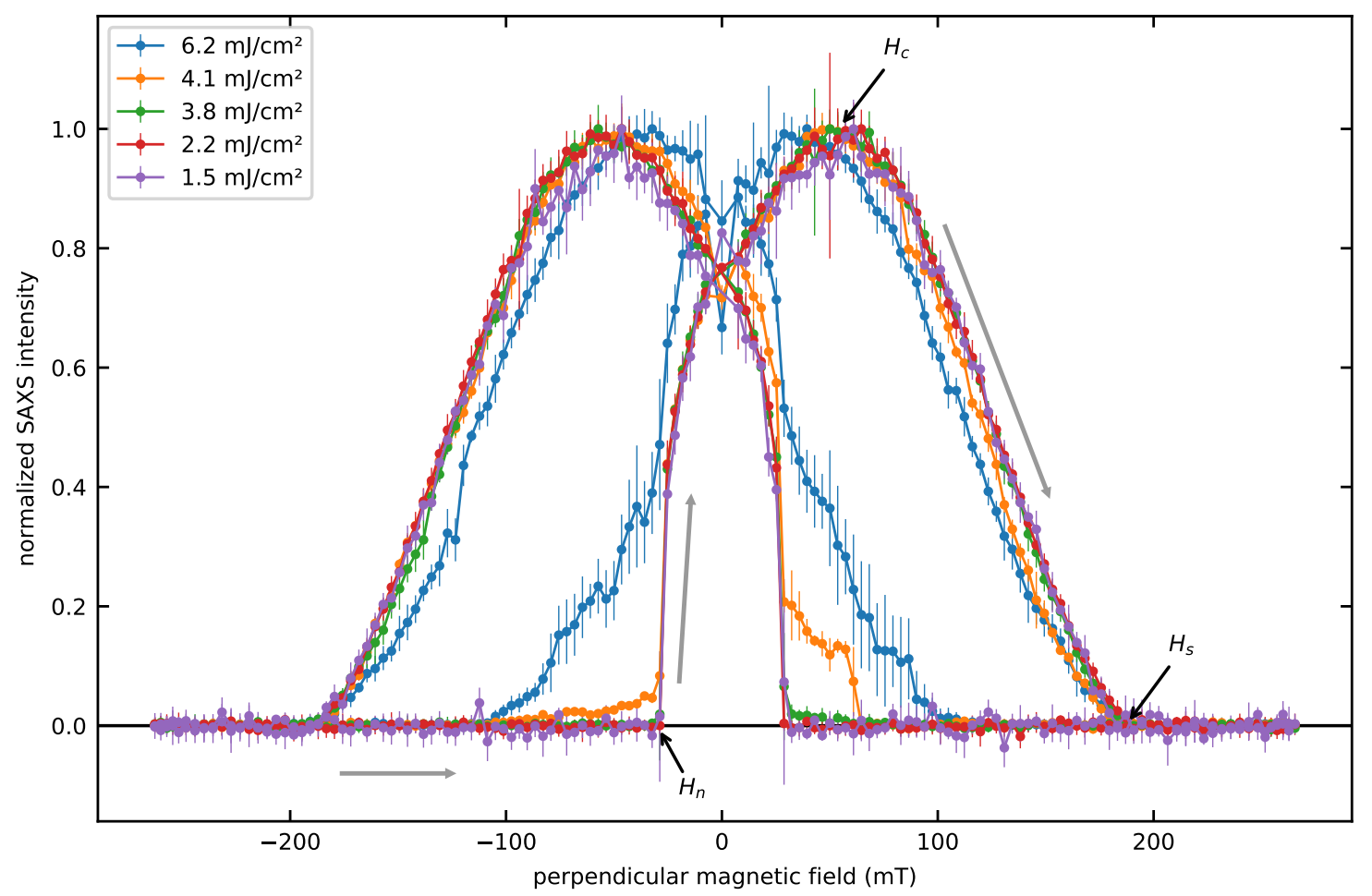

Extended Data Figure 6. Small-angle x-ray scattering during a magnetic field sweep recorded with various attenuation levels of the $x$-ray beam. The membrane sample is topographically homogeneous, which means that any scattering is due to non-uniformities in the magnetic landscape. Arrows indicate the saturation field $H_{s}$ (the field at which the scattering signal becomes zero), the nucleation field $H_{n}$ (the field at which the scattering signal starts to deviate from the background value) and the coercive field $H_{c}$ (the field at which the average magnetization is zero). Light gray arrows indicate the field sweep direction. Spectra were recorded for variable x-ray fluence by varying the transmission in a gas attenuator. The legend states the maximum peak fluence values encountered, assuming a Gaussian beam profile with a width of $30 \mu \mathrm{m}$ (FWHM). X-ray induced skyrmion nucleation and annihilation is evidenced by an increased domain nucleation field and a reduced saturation field, respectively. 

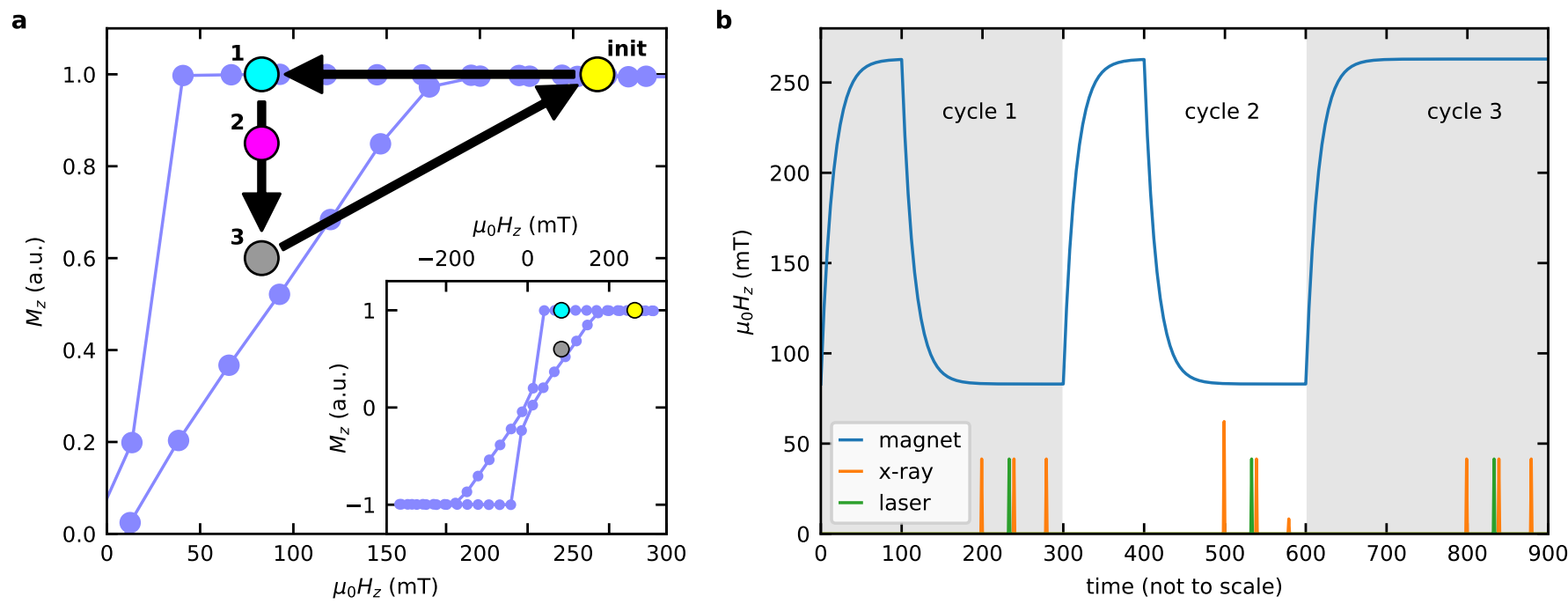

Extended Data Figure 7. Schematic of the experimental sequence. (a) Hysteresis loop of Pt/Co and illustration of the measurement cycle and static hysteresis loop of the magnetic multilayer. The measurements starts at "init" by saturating the sample $(263 \mathrm{mT})$ and reducing the field to the open hysteresis area $(83 \mathrm{mT}$, point 1$)$. The laser pulse then nucleates skyrmions (3) via a transient state (2). The inset shows the full hysteresis loop of our Pt/Co multilayer. (b) Schematic of the time traces of magnetic field, x-ray pulses, and infrared laser pulses during three successive cycles. Ideally each cycle would start with a field sweep to saturate the same and then keep the sample at remanence at $\mu_{0} H_{z}=83 \mathrm{mT}$. The pump-probe sequence consisting of three x-ray pulses and one infrared laser pulse would be applied during this stable low field time. The second and third cycle illustrate possible deviations from this scheme. As shown in the second cycle, the intensity of $\mathrm{x}$-ray pulses can vary wildly and in some cases one of the three $\mathrm{x}$-ray pulse intensities is so low that no conclusion can be drawn from the data. Moreover, as illustrated in the third sequence, the magnetic field did not always respond to the set commands, in which case no skyrmions were nucleated. Both the second and the third type of trains were rejected from the analysis. 


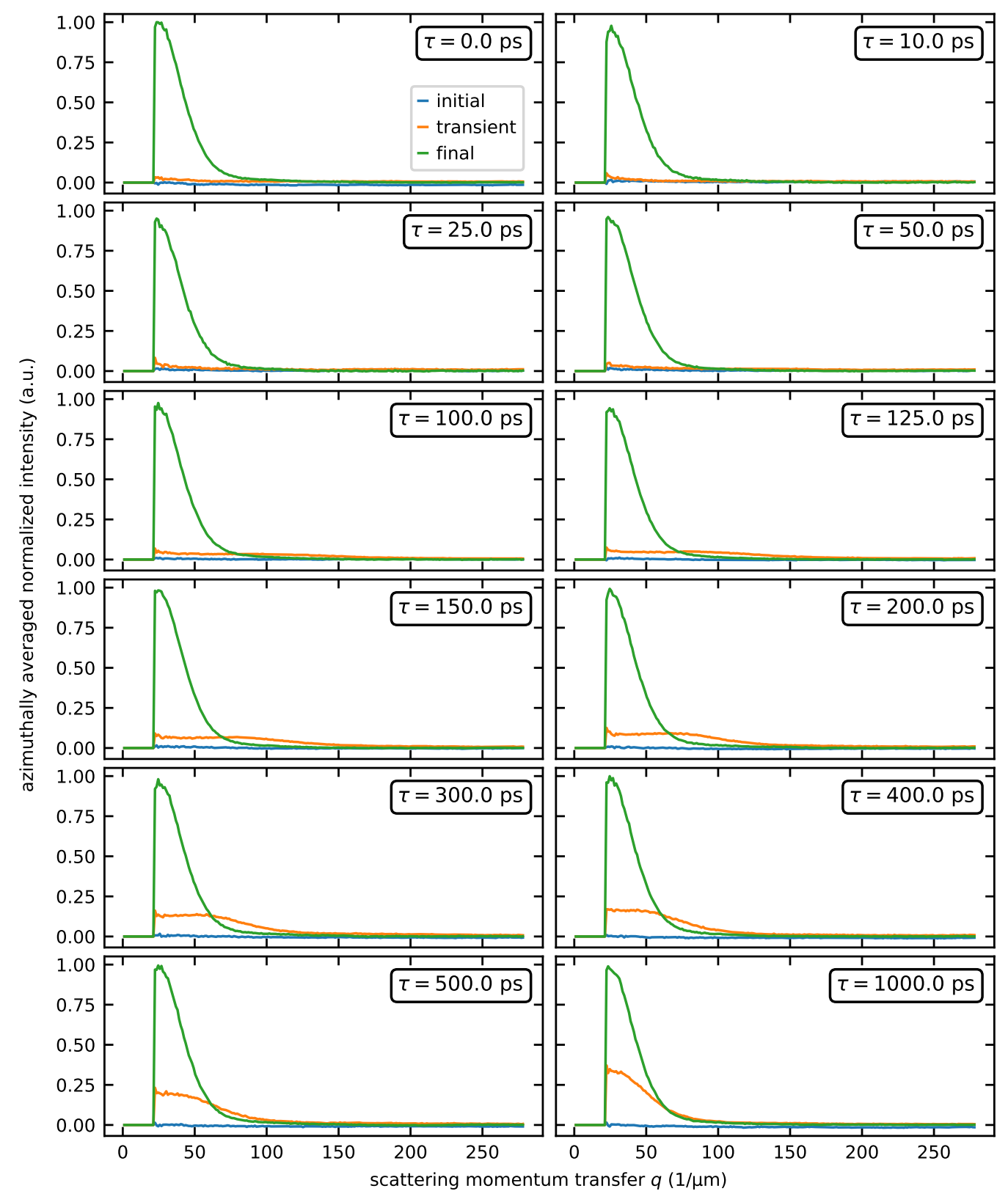

Extended Data Figure 8. Scattering spectra at all measured time delays. Orange curves show the azimuthally averaged time-dependent scattering data, which is almost constant as a function of $q$ for small delays and localizes towards smaller $q$ at later times. Blue and green lines show the corresponding initial and final state spectra, respectively. The low $q$ cutoff is due to missing pixels of the detector around the central beam. 

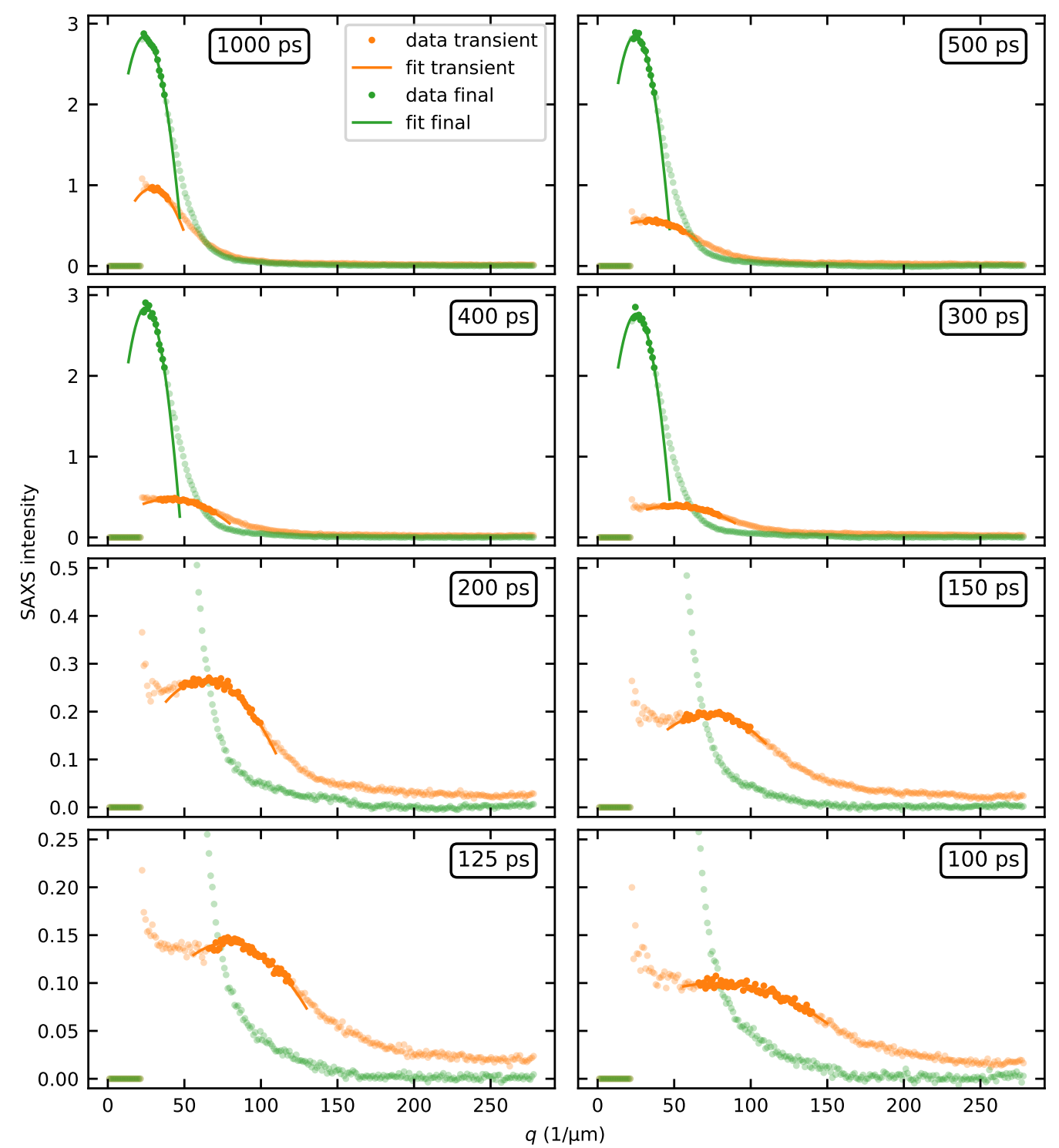

Extended Data Figure 9. Fits of the peaks of the $q$-dependent intensity distributions with a local parabola. Each panel shows the transient and final state spectra corresponding to the indicated delay. Solid lines are fits to the data. Data points considered for the fit are plotted in full contrast while all other data points are plotted with reduced contrast. The inverse of the position of the maximum of each fit, $2 \pi / q_{\text {peak }}$, is the correlation length, which is a measure of the average skyrmion distance. 


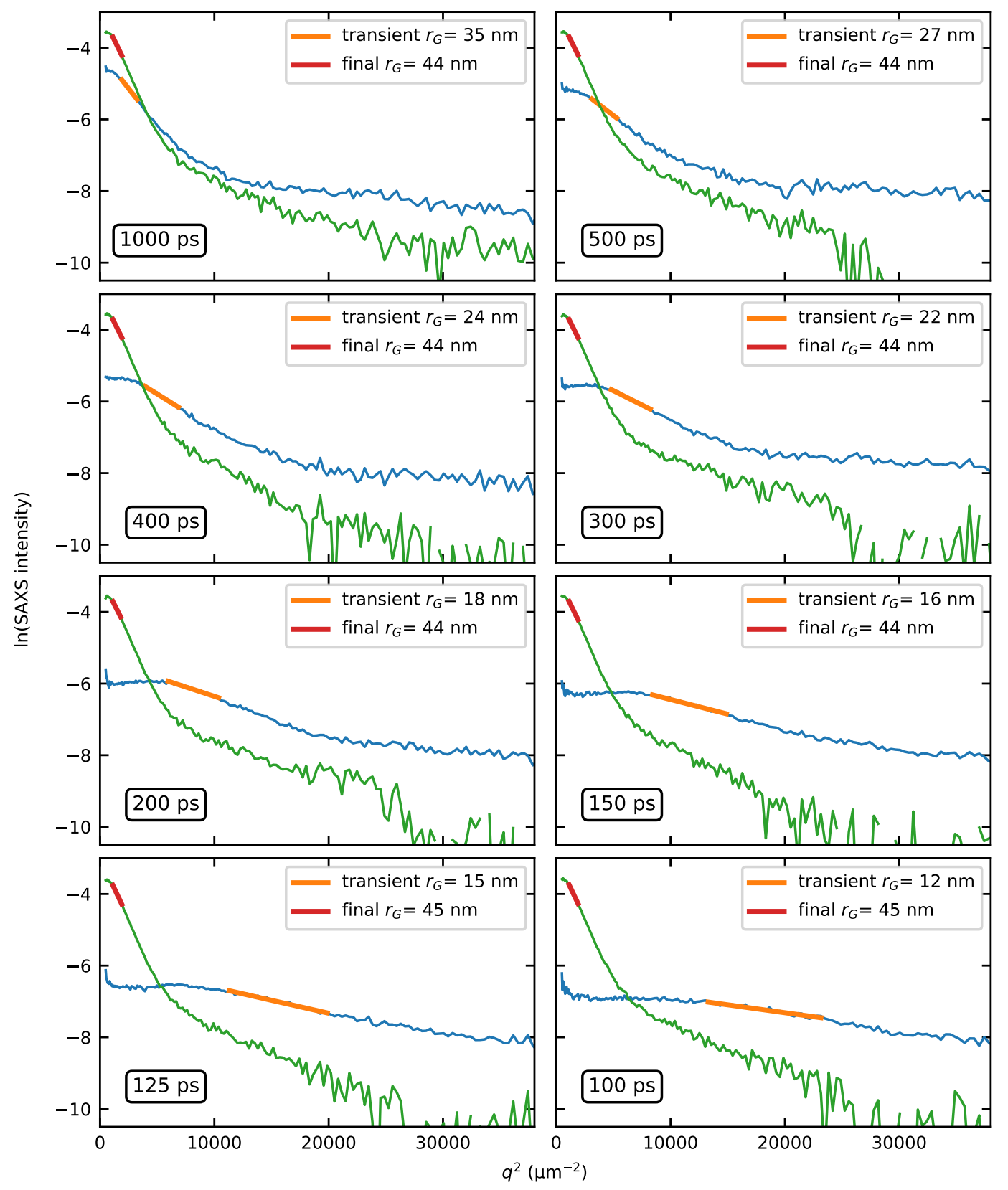

Extended Data Figure 10. Natural logarithm of the scattered intensity versus the squared scattering momentum $q^{2}$. The time delay is indicated in each panel. Each panel shows the background-corrected transient and final state spectra and the Guinier fits ${ }^{52}$ of the peak shoulders. The legend provides the radii of gyration extracted from the fits. 


\section{Supplementary Information for: Observation of fluctuation-mediated picosecond nucleation of a topological phase}

Felix Büttner*, Bastian Pfau*†, Marie Böttcher, Michael Schneider, Giuseppe Mercurio, Christian M. Günther, Piet Hessing, Christopher Klose, Angela Wittmann, Kathinka Gerlinger, Lisa-Marie Kern, Christian Strüber, Clemens von Korff Schmising, Josefin Fuchs, Dieter Engel, Alexandra Churikova, Siying Huang, Daniel Suzuki, Ivan Lemesh, Mantao Huang, Lucas Caretta, David Weder, John H. Gaida, Marcel Möller, Tyler R. Harvey, Sergey Zayko, Kai Bagschik, Robert Carley, Laurent Mercadier, Justine Schlappa, Alexander Yaroslavtsev, Loïc Le Guyarder, Natalia Gerasimova, Andreas Scherz, Carsten Deiter, Rafael Gort, David Hickin, Jun Zhu, Monica Turcato, David Lomidze, Florian Erdinger, Andrea Castoldi, Stefano Maffessanti, Matteo Porro, Andrey Samartsev, Jairo Sinova, Claus Ropers, Johan H. Mentink, Bertrand Dupé, Geoffrey S. D. Beach, and Stefan Eisebitt

${ }^{*}$ These authors contributed equally to this work

†Bastian.Pfau@mbi-berlin.de

\section{S.1 Locating the skyrmion state in the center of the hysteresis loop}

Figure $\mathrm{S} 1$ shows the average magnetization of domain states and skyrmion states in $\mathrm{Pt} / \mathrm{CoFeB} / \mathrm{MgO}$ as a function of applied field, along with a hysteresis loop measured by focused magneto-optical Kerr effect polarimetry on the same sample. The average out-of-plane magnetization of the images was determined from their average gray contrast. As suggested in Fig. 1a of the main text, the area-averaged out-of-plane magnetization $m_{z}$ of the skyrmion state is located between the two major hysteresis loops of the material. 


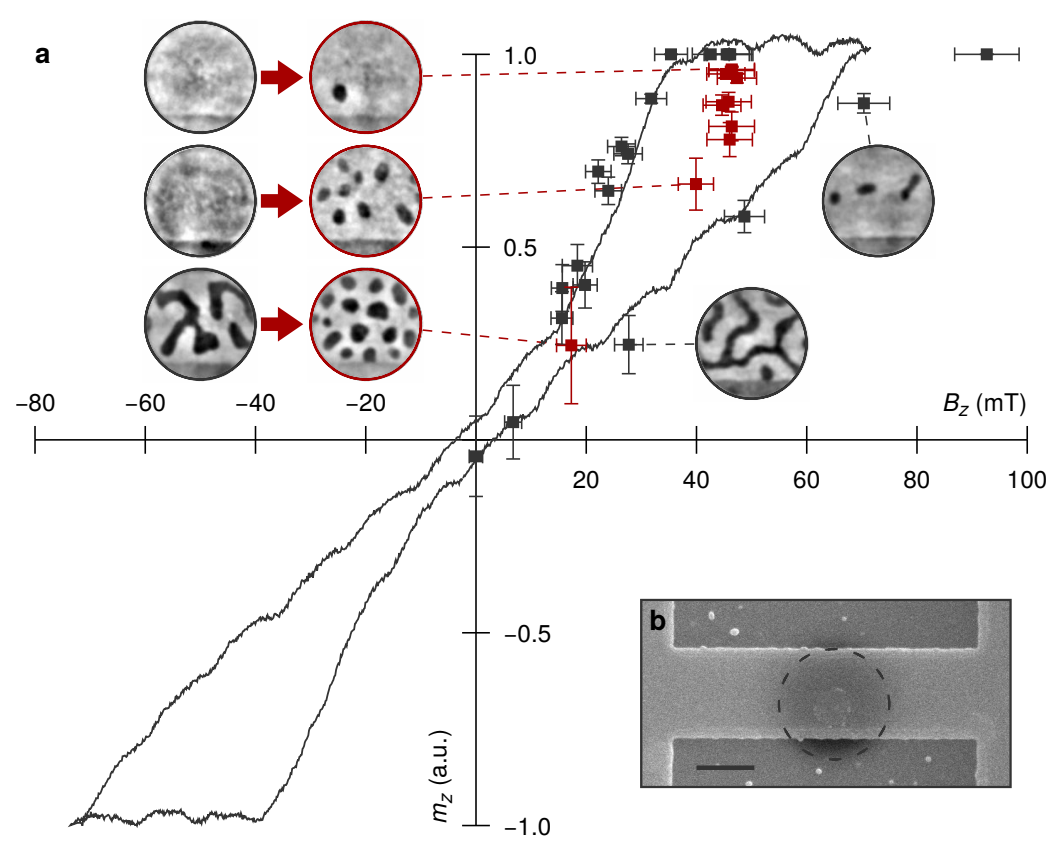

Figure S1. Field-dependent states in $[\mathrm{Pt} / \mathrm{CoFeB} / \mathrm{MgO}]_{15}$ before and after laser-induced switching within the hysteresis loop of the magnetic track. (a) Magnetic hysteresis. The solid line represents the local Kerr signal. Single data points (shown in grey and red) are extracted from x-ray images, examples of which are shown as insets. Grey data points correspond to static field cycles before laser exposure. Red data points correspond to spin states reached by laser-induced topological switching. The net magnetization in the field of view reflects the average intensity of images after binarizing to values of +1 and -1 for up and down magnetization, respectively, based on the intensity histogram of the image. The error bars (std. dev.) derive from the spatial resolution of the x-ray images. The switching fluence was $\sim 16 \mathrm{~mJ} / \mathrm{cm}^{2}$. (b) SEM image of the magnetic stripline used for the experiment in (a). The dashed circle marks the field of view with a diameter of $1 \mu \mathrm{m}$. Scale bar, $500 \mathrm{~nm}$.

\section{S.2 Evolution of stripes to skyrmions by multiple shots}

In Fig. S2 we show the images based on which Fig. 3a in the main text was constructed. One can see the gradual evolution of stripe domains to skyrmions with thousands of pulses for fluences $<14.5 \mathrm{~mJ} / \mathrm{cm}^{2}$ while at larger fluences a single pulse is sufficient to produce a pure skyrmion state. Images at each fluence were recorded in sequence, starting from a saturated state (before the first pulse).

\section{S.3 Switching threshold of Pt/Co}

Figure $\mathrm{S} 3$ shows the density of skyrmions in $\mathrm{Pt} / \mathrm{Co}$ as a function of laser fluence. As in $\mathrm{Pt} / \mathrm{CoFeB} / \mathrm{MgO}$, we find a sharp fluence threshold above which the skyrmion density is approximately constant. The threshold value of $12 \mathrm{~mJ} / \mathrm{cm}^{2}$ is comparable to $\mathrm{Pt} / \mathrm{CoFeB} / \mathrm{MgO}$. Note that for the lowest fluence used in the experiment, we observed no skyrmion nucleation in seven measurements. 


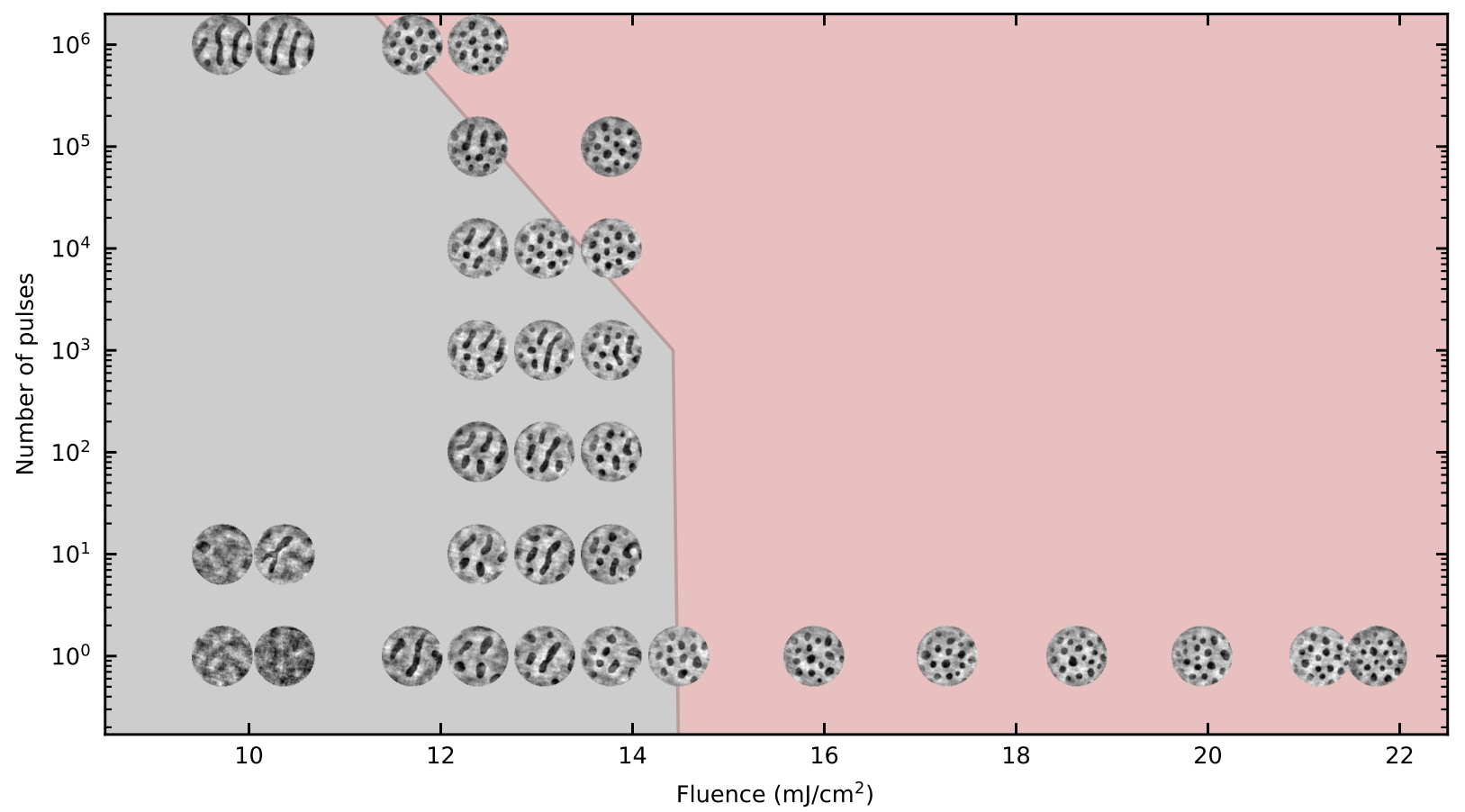

Figure S2. Magnetic textures as a function of pulse fluence and number of shots. Images correspond to the data points shown in Fig. 3a in the main text. The colored regions indicate which states we identify as skyrmions (blue) or mixed / saturated (orange). As pure skyrmion states we identify patterns of fully enclosed domains with a density that remains constant upon subsequent laser exposure. Note that equivalently, one can define those patterns as skyrmion states in which all domains are substantially circular. The equivalence is evident if one considers that any elongated domain occupies more space than a circular domain, thereby reducing the density of domains.

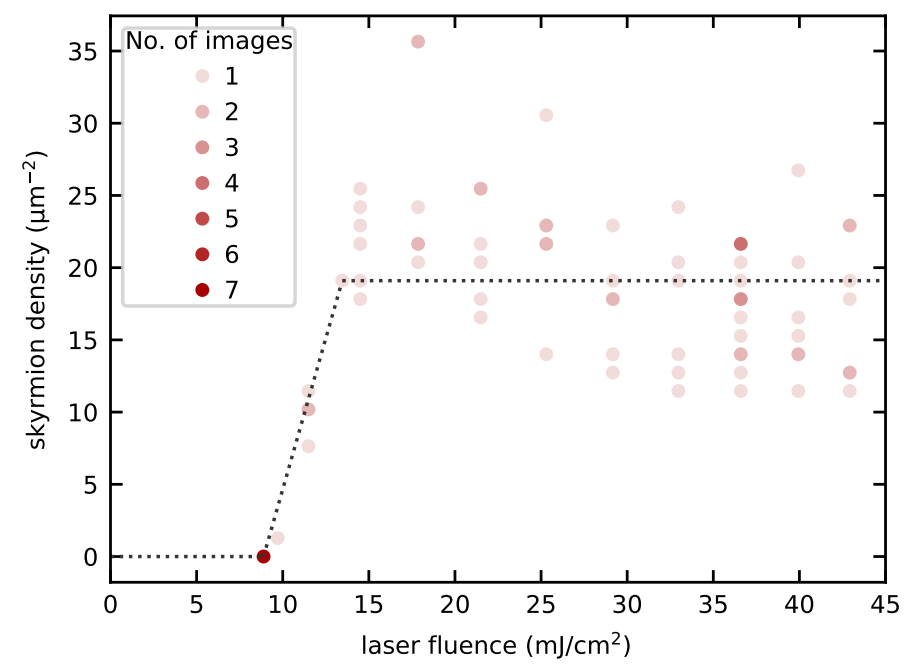

Figure S3. Fluence-dependent skyrmion density in Pt/Co. Density of skyrmions nucleated by single laser pulses from a saturated state in $\mathrm{Pt} / \mathrm{Co}$ at a bias field of $B_{z}=59 \mathrm{mT}$. Each analyzed image is represented by a semi-transparent point in the graph. Darker points thus correspond to multiple measurements of the same result, as indicated in the legend. The line is a guide to the eye. The pure skyrmion phase is characterized by the upper plateau of the skyrmion density. 


\section{S.4 Quantitative analysis of the nucleation probability distribution}

A quantitative measure of the degree of homogeneity of the probability distribution underlying an experimentally observed skyrmion distribution map is the variance of that distribution (i.e., of the number of skyrmions in each pixel) divided by the mean value of the distribution. As shown in Extended Data Figure 5, we expect that the variance-over-mean is $\sim 1$ for a homogeneous probability map, at least for the skyrmion sizes observed in the experiment and for a total skyrmion count $\leq 1000$ (for much larger numbers, the variance-over-mean starts to differ from the shot-noise expectation of 1 because one skyrmion is larger than one pixel). By contrast, for a non-homogeneous distribution, we expect the variance-over-mean to be significantly larger than 1 (Extended Data Figure 5b shows the extreme case of a nucleation probability concentrated to just one nucleation site, i.e., one pixel). The experimental results are shown in Extended Data Figures 5c,d and can clearly be assigned to a predominantly homogeneous nucleation probability map (optically induced nucleation, Extended Data Figure 5c) and to an inhomogeneous nucleation map (nucleation induced by spin-orbit torques (SOT), Extended Data Figure 5d), respectively. The SOT data is from the experiment reported in Ref. [1], details about which can be found in that reference.

\section{S.5 Fluence threshold for x-ray induced skyrmion nucleation}

X-ray free-electron laser sources have a very high x-ray pulse intensity that can be sufficient to even evaporate or ablate the sample with a single shot. During our experiment we have used x-ray pulses of $\sim 6 \mathrm{~mJ}$ pulse energy. These pulses were subsequently attenuated by the monochromator $\left(5 \times 10^{-4}\right.$ transmission), the refocusing optics ( $\sim 90 \%$ transmission) and the polarizer ( $\sim 20 \%$ transmission). Hence, in this configuration, the expected maximum energy per pulse at the sample was $\sim 55 \mathrm{~nJ}$ or, given a Gaussian x-ray spot size of $30 \mu \mathrm{m}$ (full width at half maximum, FWHM), a fluence of $5.5 \mathrm{~mJ} / \mathrm{cm}^{2}$ in the centre of the spot. This is on the same order of magnitude as the threshold to nucleate skyrmions with infrared light. For pump-probe experiments, we needed to make sure that only the pump beam (the infrared beam) was nucleating skyrmions. We therefore further attenuated the x-ray beam by a variable pressure gas attenuator. To determine the nucleation threshold, we recorded small-angle $x$-ray scattering during a magnetic field cycle with various attenuation levels of the x-ray beam. The result is shown in Extended Data Figure 6, where each data point corresponds to an average over six x-ray shots per cycle and up to four full field cycles. A non-zero scattering intensity signifies the presence of non-uniform spin structures, such as domains and skyrmions [2]. Hence, from this data, we can extract the nucleation field $H_{n}$ and the saturation field $H_{s}$, see Extended Data Figure 6. The corresponding fluences were measured on a per-pulse basis by a gas monitor detector (GMD) detecting photo-electrons and -ions. The pulse-resolved photo-electron signal was scaled to match the slower, but absolutely calibrated photo-ion signal [3]. This measurement does not include the transmission of the polarizer and refocusing optics, which are taken into account via their known transmission. The uncertainty of the final fluence value is given by the accuracy with which the $\mathrm{x}$-ray spot size is known. To measure the spot size, we used a long-range microscope to observe 
the x-ray induced fluorescence on a YAG screen in the sample plane, resulting in an uncertainty of $\sim 5 \mu \mathrm{m}$.

For a maximum $x$-ray fluence of $(4.1 \pm 1.2) \mathrm{mJ} / \mathrm{cm}^{2}$ or higher (10\% gas attenuator transmission), we observe that, compared to lower x-ray intensities, $H_{n}$ becomes larger (domains nucleate earlier when coming from saturation) and $H_{s}$ becomes smaller (i.e., the last domains annihilate easier). This is consistent with the narrowing of the hysteresis loop after laser exposure shown in Fig. S1. We therefore conclude that above this threshold we see x-ray induced nucleation and annihilation of skyrmions or reversed domains. At $(3.8 \pm 1.1) \mathrm{mJ} / \mathrm{cm}^{2}(7.5 \%$ transmission $)$ and below, both $H_{n}$ and $H_{s}$ are no longer x-ray fluence dependent. We therefore choose to operate at $7.5 \%$ transmission. Note that, due to the large shot-to-shot intensity fluctuations, exceptionally high intensity pulses may still be above the nucleation threshold even at $7.5 \%$ transmission. However, during the pump-probe experiments, we have stored the camera data of each shot separately and unusually intense x-ray pulses were not considered in the analysis (see Section S.7).

Note that the threshold fluences for x-ray induced switching are very similar to the infrared regime, supporting our interpretation that fast heating is driving the transition without particular sensitivity to the means by which the heating is achieved. However, considering the enormous pulse-to-pulse variation, our estimates of the x-ray fluences based on the optical density of the gas attenuator is highly approximate. Moreover, whether the x-ray nucleated spin textures are actually topological remains to be confirmed. While not being fully conclusive, our observations point to the possibility of x-ray pump - x-ray probe experiments, where larger penetration depth, element-selective excitations, and a potentially much smaller focus are only some of the prospective benefits compared to laser-driven excitations.

\section{S.6 Masking of the time-resolved scattering camera images}

We have used a DSSC detector $[4,5]$ comprising 16 rectangular pixel array modules arranged in a square grid. Each quadrant of this grid was covered by an Al filter to block the infrared beam. Moreover, there was a finite spacing between these modules. After mapping the data to this geometry, a camera image as shown in Fig. S4a is obtained (which shows an image without any x-ray scattering). Note that small uncertainties in the panel geometry arise from the fact that the detector is assembled from separate modules. While such uncertainties would impose a problem for imaging, they are irrelevant for our scattering analysis.

We have applied a mask to all recorded images to remove artifacts. First, we notice that there is still signal from the infrared beam very close to the centre of the detector. These regions were masked and removed. In addition, we have masked all pixels below the frame of the Al filter since these pixels did not detect any x-rays and only contributed to the noise. Finally, we have masked dead pixels. The result is shown in Fig. S4b. The same mask was applied to all frames. Note that masked pixels were ignored when normalizing the azimuthal integral of the scattered intensity. 

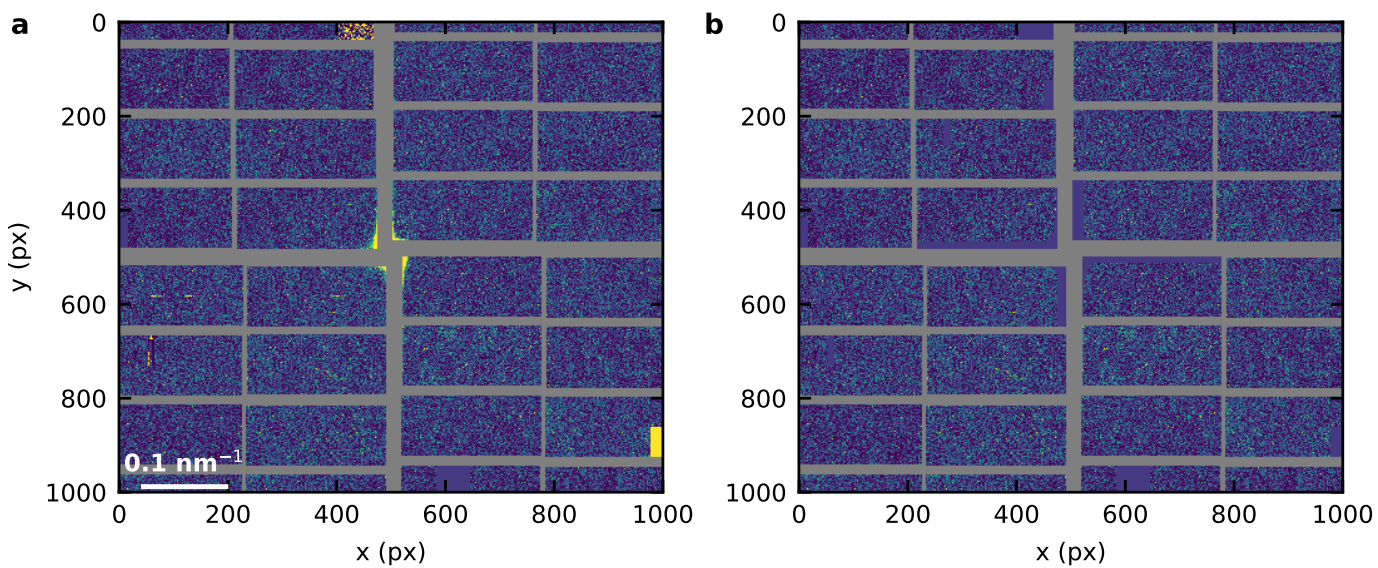

Figure S4. Masking of DSSC camera images for time-resolved x-ray scattering. (a) Cropped raw camera image after arranging the pixels according to the physical detector geometry and subtracting a dark frame. The image was recorded while exposing the sample with infrared light but not with x-rays. (b) Same image as in (a) after applying the mask filter. Note that images appear stretched along the $x$ direction due to an hexagonal arrangement of the pixels, which leads to a pixel periodicity of $236 \mu \mathrm{m}$ and $204 \mu \mathrm{m}$ in $x$ and $y$ direction, respectively. This distortion was considered in our analysis.

\section{S.7 Frame selection of time-resolved scattering data}

The desired sequence of our time-resolved scattering experiments is illustrated in Extended Data Figure 7. In this scheme, a sequence starts by saturating the sample, followed by the reduction of the field and the exposure of the sample with a train of three x-ray pulses and one infrared laser pulse just before the second $\mathrm{x}$-ray pulse. The field cycle ensures that the sample is prepared every time in a metastable uniform state where skyrmion nucleation is possible by ultrafast heating. However, our experiments were conducted during the early user startup phase of the European XFEL that normally delivers $\mathrm{x}$-ray pulse trains at $10 \mathrm{~Hz}$ repetition frequency. This was too fast to cycle the magnetic field and on-demand request of x-ray pulses and magnetic fields was not fully implemented. We therefore have applied filters to select only those trains that actually represent the desired experimental sequence. Specifically, we considered only trains where all of the following conditions were fulfilled:

1. The train includes exactly three $\mathrm{x}$-ray pulses.

2. The previous train does not include any x-ray pulses.

3. All x-ray pulses in the train were of significant intensity to give a statistically meaningful scattering pattern (see Fig. S5).

4. The intensity of the first x-ray pulse in the train was below a threshold to exclude skyrmion nucleation by the $\mathrm{x}$-ray pulse (see Fig. S5).

5. The initial state is magnetically uniform (integrated scattering intensity below threshold, see Fig. S6). This criterion makes sure that the state was properly reset by the magnetic field. 
6. The final state is a skyrmion state (integrated scattering intensity above threshold, see Fig. S6). This selection is required because the magnetic field for some pump-probe trains was still high, preventing skyrmion formation.

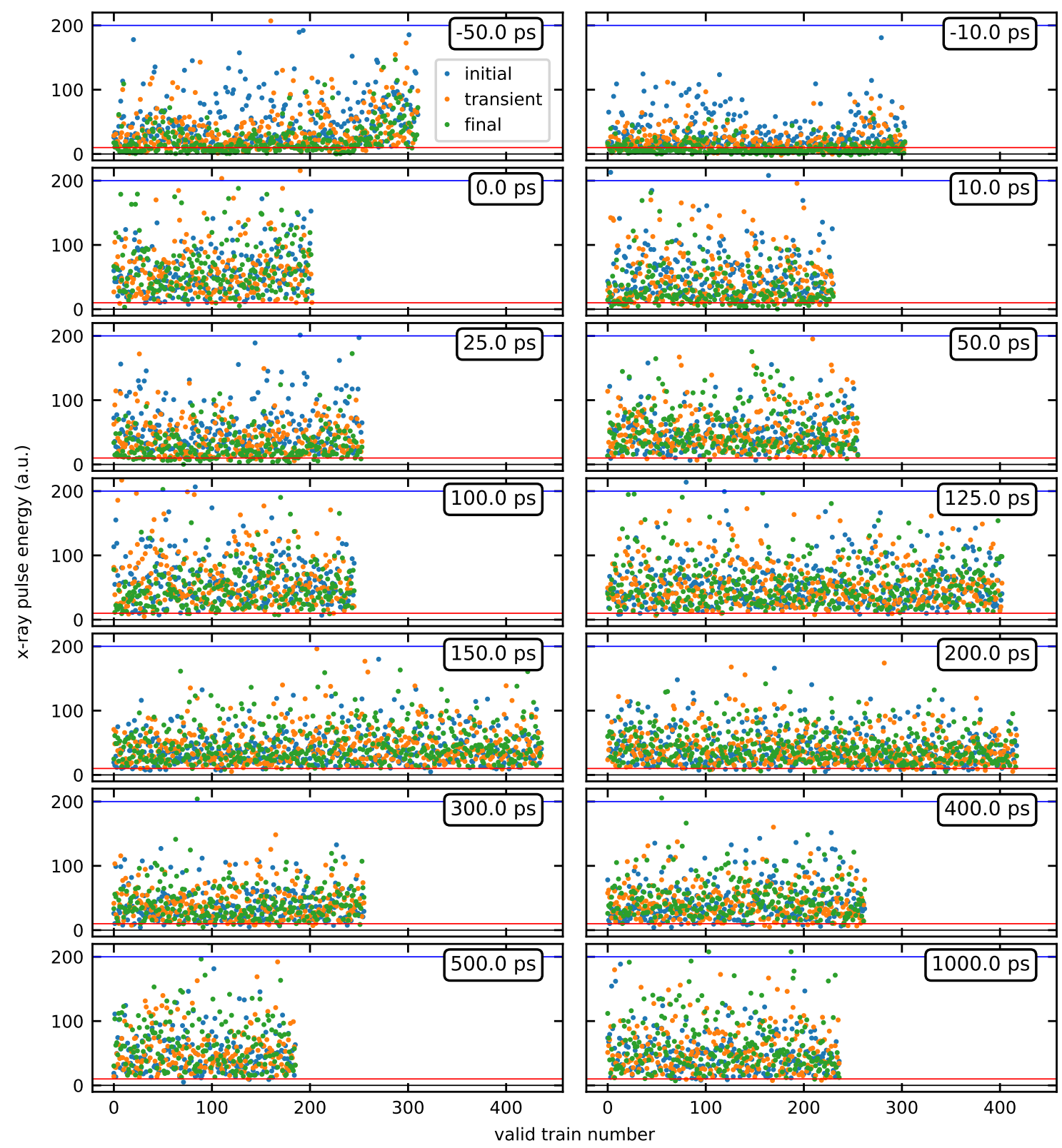

Figure S5. Threshold selection of trains by x-ray pulse intensity. Each panel shows the x-ray pulse intensity as measured with a gas intensity monitor after the monochromator in the beamline. We select only trains where the intensity of all three x-ray pulses is above the detection limit (red line) and where the intensity of the first $\mathrm{x}$-ray pulse is below the empirical $\mathrm{x}$-ray skyrmion nucleation threshold (blue line; see also Extended Data Figure 6). 


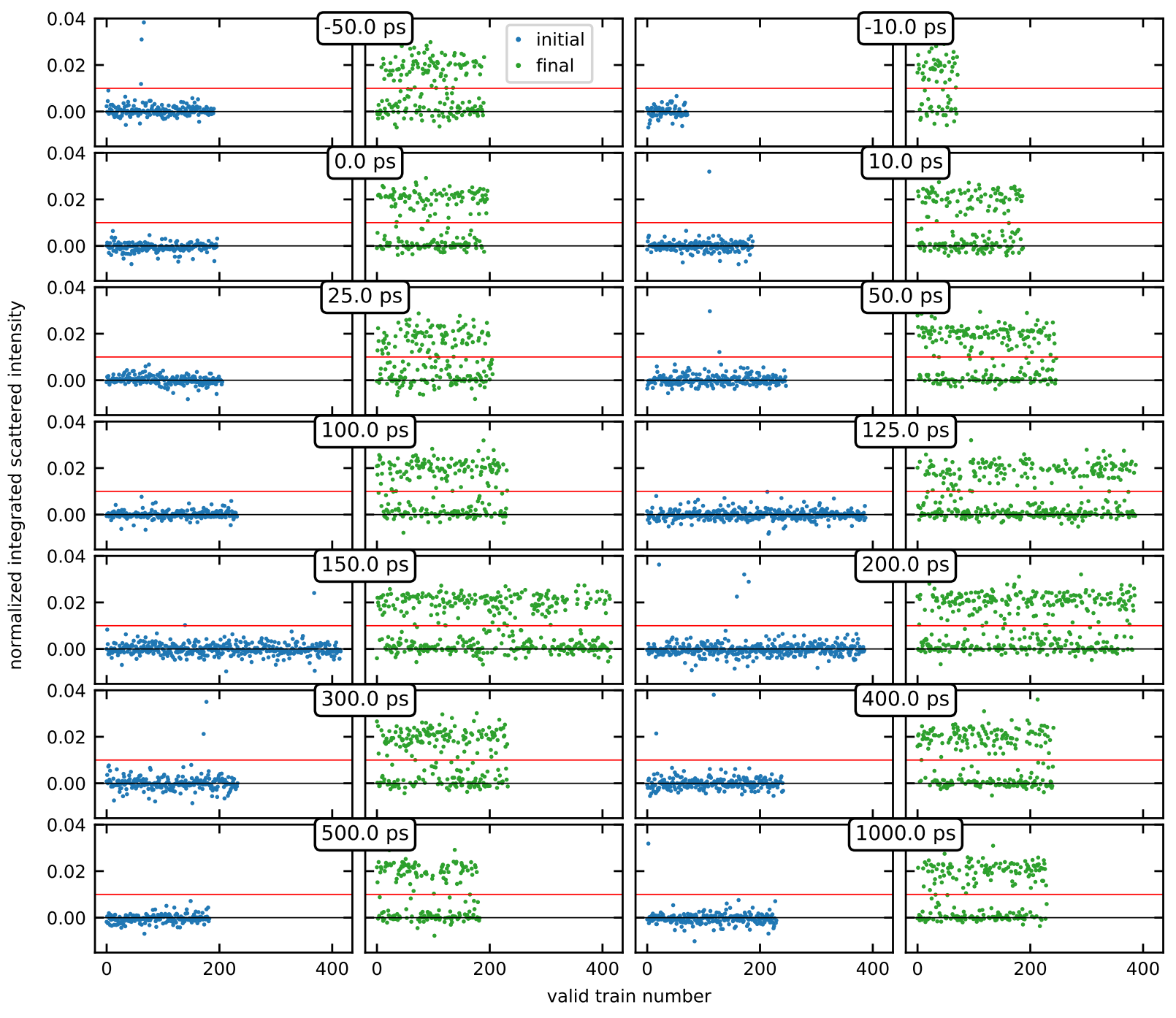

Figure S6. Threshold selection of trains by normalized scattered intensity in the initial and final state. For each time delay we plot the normalized integrated scattered intensity of all pulse trains that have passed tests 1-4. We notice a binary distribution in the final state intensity, which represents the case of skyrmion nucleation (high value) and no skyrmion nucleation (low value). We select only those trains where the final state scattered intensity is high, i.e., where skyrmions were nucleated. Below-threshold data represents trains recorded at a high magnetic field where skyrmion nucleation was not possible. In addition, we discard the few trains where the initial state intensity is above the threshold, i.e., where the skyrmion state was not successfully reset by the magnetic field. 


\section{S.8 Control measurement: constant initial and final state scattering}

Each pump-probe measurement was accompanied by a control measurement of the initial state $(60 \mu \mathrm{s}$ before the laser pulse) and the final state $(60 \mu$ s after the laser pulse). The $I(q, t)$ intensity maps of these control data are shown in Figs. S7 and S8 (where $t$ is the time delay of the corresponding pump-probe data). The experiment was conducted in descending sequence starting from large time delays (with the exception of $t=0 \mathrm{ps}$, which was recorded first). The initial state data reveals a slowly increasing background count towards later measurement times (earlier pump-probe delays). This background was subtracted from the transient and final state data. After background correction, the final state scattering is almost perfectly constant as a function of $t$, confirming that the transient state time-dependent data is in fact only related to transient changes of the magnetic pattern.

\section{S.9 Robustness of the fit of the total integrated intensity}

The total integrated intensity in Fig. $4 \mathrm{~d}$ in the main text was obtained by summing the intensities of all pixels up to a cutoff $q_{\max }$ value. The reason for the cutoff is that very high $q$ pixels contribute more noise than data, making the extracted total intensity less meaningful. To confirm that the physical interpretation does not depend on our choice of the integration cutoff, we plot in Fig. S9 the fit parameters for a broad range of cutoff values. While the inflection point of $I(t)$ shows considerable variation, both the timescale of equilibration and the saturation at $300 \mathrm{ps}$ are robust with respect to the integration cutoff.

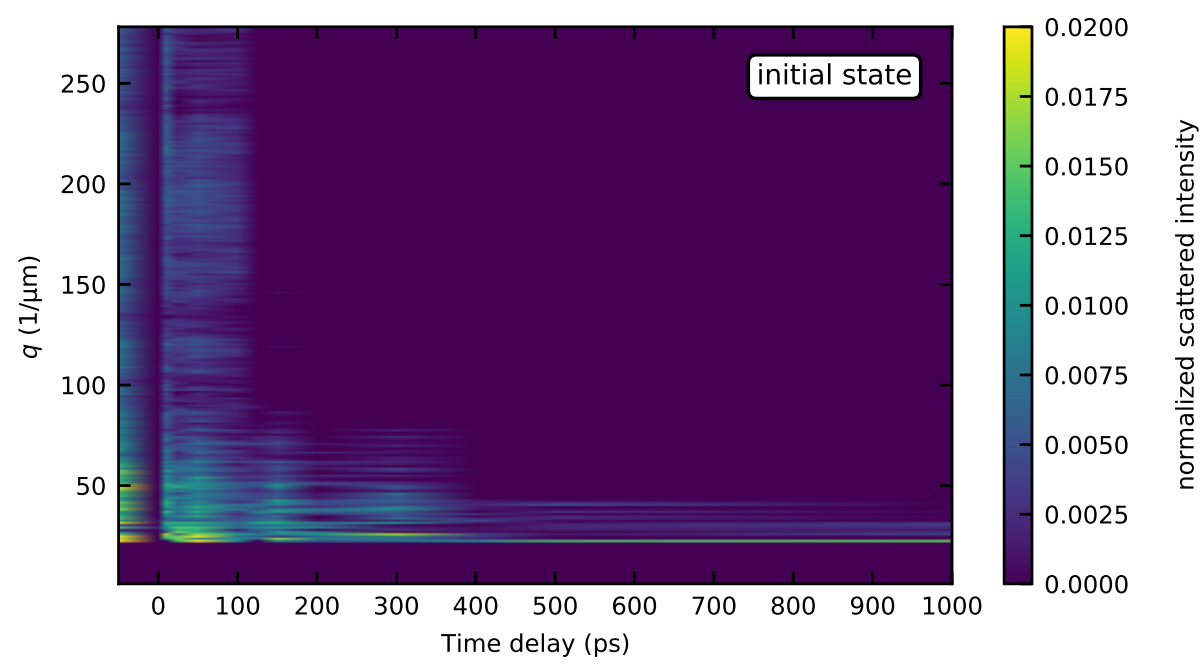

Figure S7. Initial state $q$-dependent scattering as a function of delay of the transient state $x$-ray pulse. The small signal at small time delays is due to thermal drift of the readout noise. This offset was subtracted from the transient and final state data. 


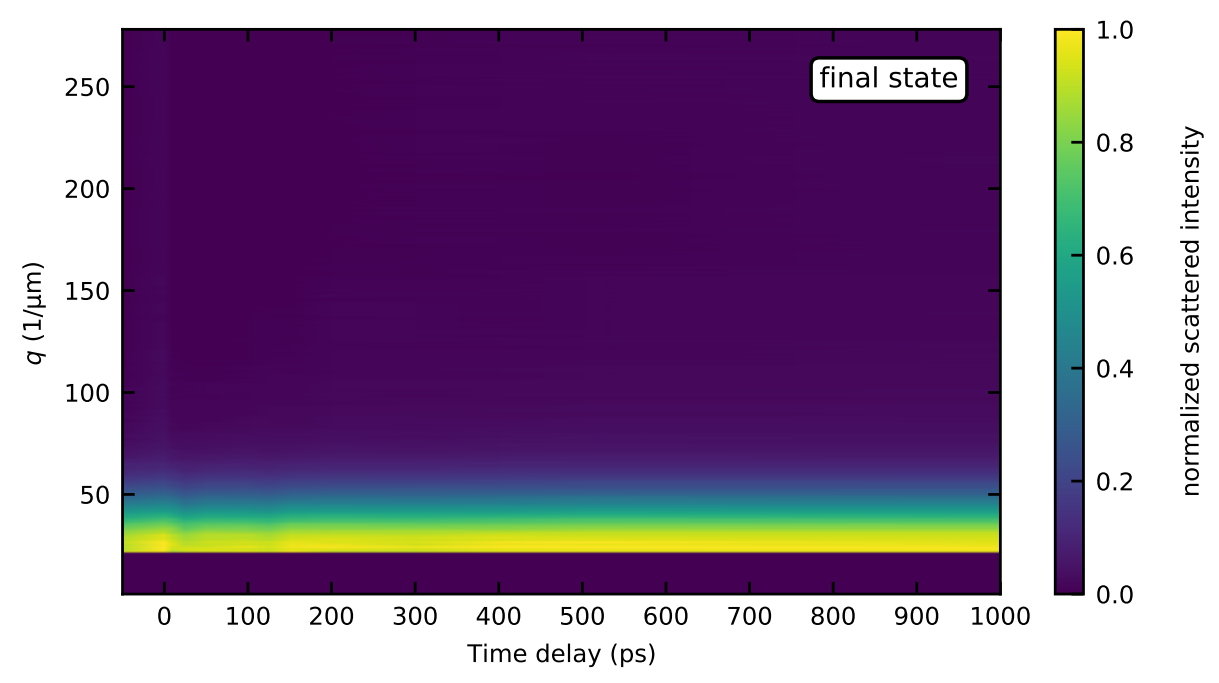

Figure S8. Final state $q$-dependent scattering as a function of delay of the transient state x-ray pulse. The signal is flat, indicating stable pump-probe conditions during our experiment.

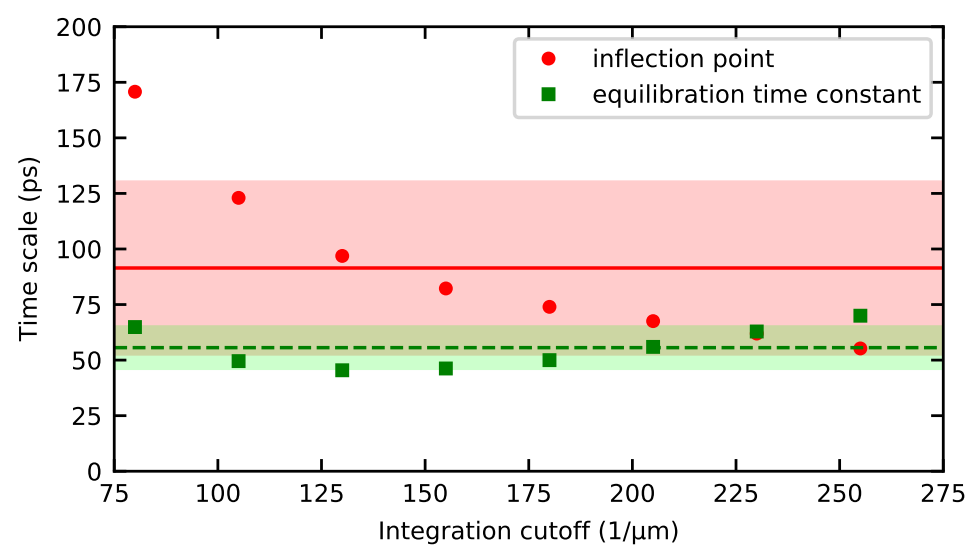

Figure S9. Total intensity fit results as a function of integration cutoff. Data points show the inflection point (time delay where the change of total intensity is largest) and the exponential equilibration time constant extracted from a logistic function fit to the integrated intensity for variable cutoff value of the integration. The dashed lines represent the averages and the bands indicate the one standard deviation error bar on each side of the average. 


\section{S.10 Analysis of scattering spectra}

In Extended Data Figure 8 we show the complete set of scattering spectra, where all previously discussed corrections have been applied. The transient data (orange) is initially almost constant as a function of $q$, i.e., there is significant intensity scattered to high angles. At later times, transient spectra exhibit a pronounced intensity maximum at low momentum transfer. For comparison, we also show the spectra of the initial (blue) and final state (green). We do not detect any scattering in the initial state and all final state spectra are identical within the experimental shot noise (see also Figs. S7 and S8). At $1 \mathrm{~ns}$ of time delay between pump and probe the scattering function of the transient state is already similar to the one of the final state, even though it has not yet reached the full intensity.

Our scattering data can be seen as a superposition of two main contributions: scattering off a single skyrmion nucleus (the so-called form factor), which is characteristic of its size and shape, and scattering off the ensemble of skyrmions (the structure function), which encodes the positions of skyrmions relative to each other. Given the high density of skyrmions, both the size and the relative distance are of similar order of magnitude and the scattering signals strongly overlap. Lacking a reliable quantitative model of the transient spin textures in our experiment, those contributions cannot be disentangled analytically. Instead, we obtain approximate estimates of the mean skyrmion distance and size from the most distinctive features of our recorded intensity spectra, namely the position of the intensity maximum $q_{\text {peak }}$ and a section of the high- $q$ shoulder of this maximum where $\ln (I(q))$ decreases linearly with $q^{2}$, respectively. These features can be identified starting with a delay of $100 \mathrm{ps}$ after the pulse.

The intensity maximum reflects the correlation in the positions of the skyrmions and, thus, acts as a measure for the mean distance between the skyrmions. We therefore calculate the average skyrmion distance via $2 \pi / q_{\text {peak }}$. To determine $q_{\text {peak }}$, we fit the $I(q)$ spectra near the peak with a parabola $I(q)=$ $a\left(q-q_{\text {peak }}\right)^{2}+c$, see Extended Data Figure 9. Note that the same results were obtained when fitting with a Voigt function. The temporal evolution of the fit parameters is shown in Fig. S10.

A size estimate of the skyrmion nuclei is extracted from a Guinier fit. The Guinier approximation predicts for the scattering of dilute particles for small $q$ (typically $r_{G} q<1$ ):

$$
\ln (I(q))-\ln (I(0)) \simeq-\frac{1}{3} r_{G}^{2} q^{2}
$$

Here, $r_{G}$ is called the "radius of gyration" of the scattering particle.

In Extended Data Figure 10 we show $\ln (I)$ vs. $q^{2}$ plots of the SAXS spectra. At the high- $q$ side of the intensity maximum, we find a regime in which this graph is linear, i.e., where $\ln (I(q))$ is directly proportional to $q^{2}$. To obtain $r_{G}$, we perform a linear regression in this region. Following the recommendations in [6], we iteratively refine the fit range and use an upper limit of $r_{G} q \leq 2.0$ where the Guinier fit is still meaningful with acceptable error. For our data, the lower limit for the fit is given by the scattering intensity maximum due to the inter-particle correlation. We, thus, define the fit range to $1.5 \leq r_{G} q \leq 2.0$, consistently for all delay times.

The radius of gyration is a direct measure of the size of the scattering particles (i.e., the skyrmions) 
which we assess by their mean diameter as $d \simeq 2 \chi r_{G}$ [6]. The proportionality factor $\chi$ depends on many parameters, such as the shape, size distribution and spatial distribution of the particles as well as the fit range (a fit at larger $r_{G} q$ results in larger error of $\chi$ ). The most accurate estimate of $\chi$ is obtained in the final state, where direct real-space information is available. A histogram of the skyrmion size distribution is presented in Fig. S11 revealing a mean skyrmion diameter of $73 \mathrm{~nm}$, corresponding to $\chi=0.83$. With a known value of $\chi$, our method is very precise, as evidenced by the very small variation of the final state mean diameters shown in Fig. $4 \mathrm{~d}$ of the main paper. To account for the fact that shape and distribution of the skyrmions in the intermediate state likely deviate from the final state properties in an unknown way, we use the same $\chi$ for the intermediate state conversion but add a systematic error of $30 \%$, which is on the high side of expected uncertainties corresponding to our fit range [6].
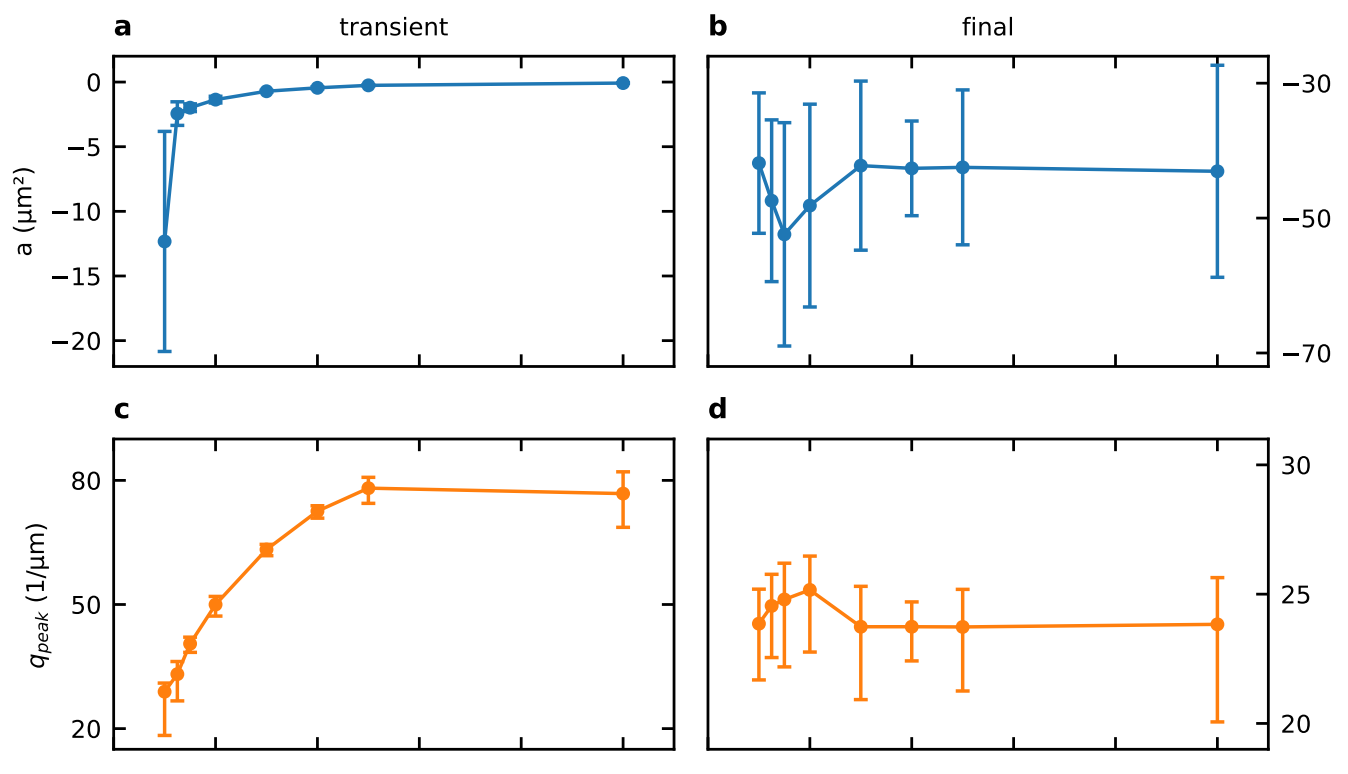

d
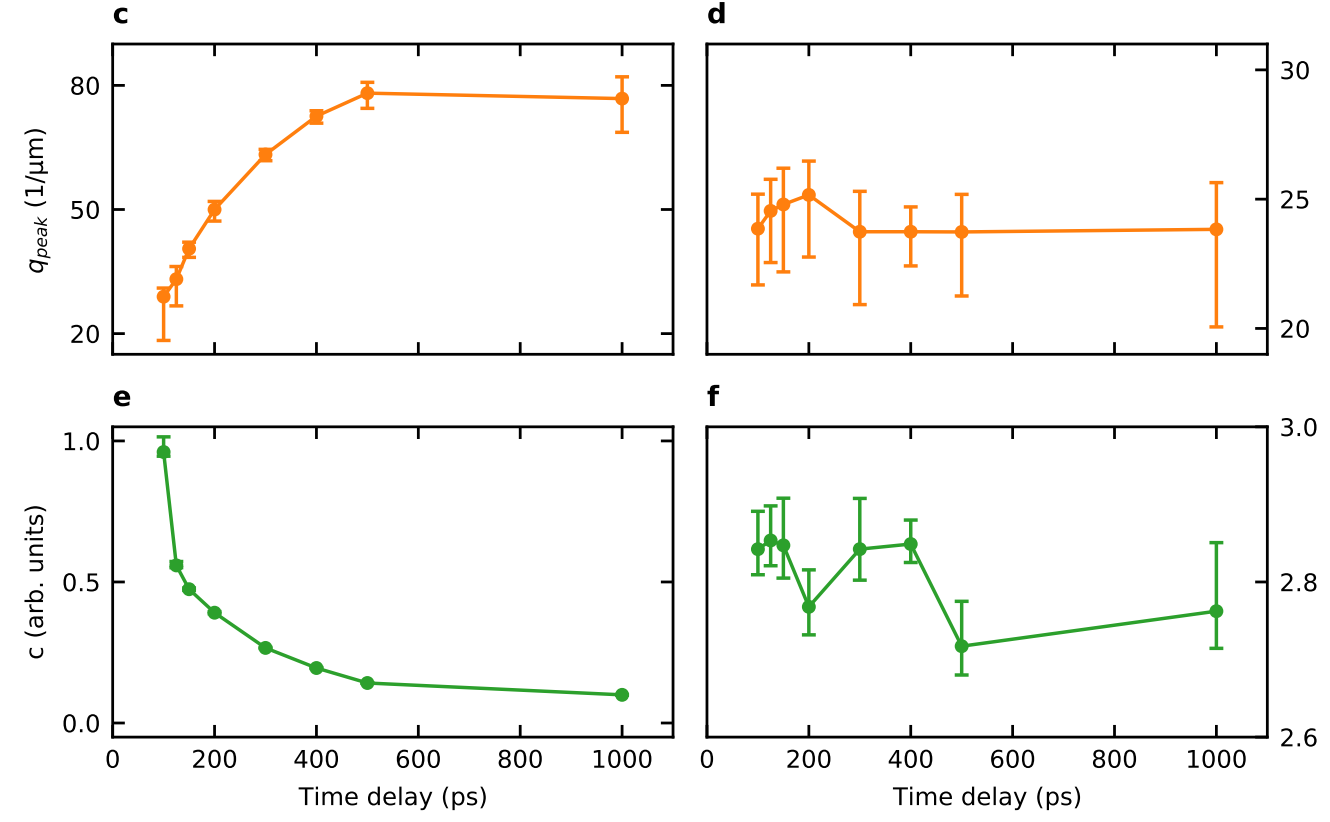

Figure S10. Fit parameters of the peak fit as a function of pump-probe delay.

\section{S.11 Supplementary Video}

The video shows four panels. On top is a plot of the total topological charge and the bath temperature as a function of time. A black vertical line indicates the current time of the simulation. Below are three panels, showing the normalized out-of-plane magnetization, the local topological charge density, and 


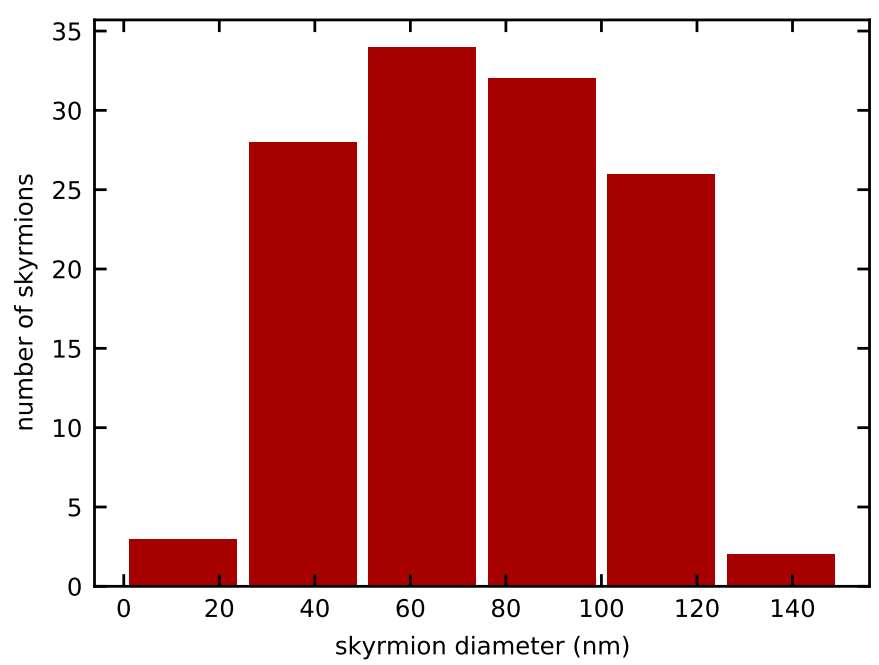

Figure S11. Histogram of the skyrmion size distribution in Pt/Co in the final state The histogram was compiled from six holography images recorded using the same conditions as in Fig. 2c, top row, of the main paper.

a low-pass filtered version of the local topological charge density, where the filter size of the low-pass filter in reciprocal unit cells is indicated in the filtered image.

\section{References}

[1] Büttner, F. et al. Field-free deterministic ultrafast creation of magnetic skyrmions by spin-orbit torques. Nature Nanotechnology 12, 1040-1044 (2017).

[2] Hellwig, O., Maat, S., Kortright, J. B. \& Fullerton, E. E. Magnetic reversal of perpendicularlybiased Co/Pt multilayers. Physical Review B 65, 144418 (2002).

[3] Grünert, J. et al. X-ray photon diagnostics at the European XFEL. Journal of Synchrotron Radiation 26, 1422-1431 (2019).

[4] Porro, M. et al. Development of the DEPFET Sensor With Signal Compression: A Large Format X-Ray Imager With Mega-Frame Readout Capability for the European XFEL. IEEE Transactions on Nuclear Science 59, 3339-3351 (2012).

[5] Hansen, K. et al. Qualification and Integration Aspects of the DSSC Mega-Pixel X-Ray Imager. IEEE Transactions on Nuclear Science (2019).

[6] Feigin, L. A. \& Svergun, D. I. Structure Analysis by Small-Angle X-Ray and Neutron Scattering (Plenum Press, New York, NY, USA, 1987). 